\title{
CDKN2B upregulation prevents teratoma formation in multipotent fibromodulin-reprogrammed cells
}

\author{
Zhong Zheng, ${ }^{1}$ Chenshuang Li, ${ }^{1}$ Pin Ha, ${ }^{1}$ Grace X. Chang, ${ }^{2}$ Pu Yang, ${ }^{3}$ Xinli Zhang, ${ }^{1}$ Jong Kil Kim, ${ }^{1}$ Wenlu Jiang, ${ }^{1,3}$ Xiaoxiao Pang,,${ }^{1,4}$ \\ Emily A. Berthiaume, ${ }^{2}$ Zane Mills, ${ }^{5}$ Christos S. Haveles, ${ }^{2}$ Eric Chen, ${ }^{1}$ Kang Ting ${ }^{1,6}$ and Chia Soo ${ }^{6}$ \\ 'Division of Growth and Development, School of Dentistry, and 2David Geffen School of Medicine, UCLA, Los Angeles, California, USA. ${ }^{3}$ State Key Laboratory of Oral Diseases, Department of Orthodontics, \\ West China School of Stomatology, Sichuan University, Chengdu, China. ${ }^{4}$ Chongqing Key Laboratory of Oral Diseases and Biomedical Sciences, Stomatology Hospital of Chongqing Medical University, \\ Chongqing, China. ${ }^{5}$ Department of Ecology and Evolutionary Biology, and ${ }^{6}$ Division of Plastic and Reconstructive Surgery and Department of Orthopaedic Surgery and the Orthopaedic Hospital Research \\ Center, UCLA, Los Angeles, California, USA.
}

\begin{abstract}
Tumorigenicity is a well-documented risk to overcome for pluripotent or multipotent cell applications in regenerative medicine. To address the emerging demand for safe cell sources in tissue regeneration, we established a novel, protein-based reprogramming method that does not require genome integration or oncogene activation to yield multipotent fibromodulin (FMOD)-reprogrammed (FReP) cells from dermal fibroblasts. When compared with induced pluripotent stem cells (iPSCs), FReP cells exhibited a superior capability for bone and skeletal muscle regeneration with markedly less tumorigenic risk. Moreover, we showed that the decreased tumorigenicity of FReP cells was directly related to an upregulation of cyclindependent kinase inhibitor 2B (CDKN2B) expression during the FMOD reprogramming process. Indeed, sustained suppression of $C D K N 2 B$ resulted in tumorigenic, pluripotent FReP cells that formed teratomas in vivo that were indistinguishable from iPSC-derived teratomas. These results highlight the pivotal role of CDKN2B in cell fate determination and tumorigenic regulation and reveal an alternative pluripotent/multipotent cell reprogramming strategy that solely uses FMOD protein.
\end{abstract}

\section{Introduction}

Local endogenous stem/progenitor cells are often unable to adequately reestablish tissue integrity and function after largevolume tissue loss from trauma, degenerative pathologies, or aging. In these cases, exogenous replacement cells derived from the stem or progenitor cells are necessary to restore lost tissue (1). Unfortunately, despite decades of investigation, isolation and/or generation of safe and readily available regenerative cell sources remain major challenges. In particular, the tumorigenicity risks are not only posed by teratomas formed by embryonic stem cells (ESCs) and induced pluripotent stem cells (iPSCs), but also include the tumor-supporting and/or formation potential of mesenchymal stem cells (MSCs) (2, 3), the main cell source for regenerative medicine used in the last few decades (4).

To address the emerging demand for safe and easily obtainable cell sources for tissue regeneration, we previously established an alternative strategy using continuous recombinant human fibromodulin (FMOD) stimulation under serum-free conditions to reprogram human dermal fibroblasts into multi-

Authorship note: $Z Z$ and $C L$ are co-senior authors and contributed equally to this work. Conflict of interest: $\mathrm{ZZ}, \mathrm{KT}$, and CS are inventors on fibromodulin-related patents assigned to UCLA (ZL 201180023880.9; US 9,409,963 B2; US 9,885,014 B2; US 9,549,954 B2; US and 10,190,097 B2). ZZ, KT, and CS are founders of Scarless Laboratories Inc., which sublicenses fibromodulin-related patents from the University of California Board of Regents, which also holds equity in the company. ZZ and CS are also former and current officers, respectively, of Scarless Laboratories Inc. Copyright: (c) 2019, American Society for Clinical Investigation.

Submitted: September 18, 2018; Accepted: May 21, 2019; Published: July 15, 2019 Reference information: J Clin Invest. 2019;129(8):3236-3251.

https://doi.org/10.1172/JCl125015. potent cells (5). FMOD reprogramming shares several technological conveniences seen with iPSC generation. Both methodologies (a) offer freedom from the ethical and logistical constraints overshadowing ESC generation; (b) reprogram autologous cells, which significantly reduces the risk of immunogenic rejection; and (c) transform human dermal fibroblasts, a cell type that can be easily obtained and expanded from a skin biopsy (6), which may be preferred over the invasive harvest procedures required to obtain sufficient MSCs. Using this technology, dermal fibroblasts isolated from donors of different ages and sexes have been successfully reprogrammed into a multipotent state $(5,7)$. The resultant dome-shaped, clustered FMOD-reprogrammed (FReP) cells are easily separated from the surrounding spindle-shaped, monolayer FReP-basal cells with a xeno-free and enzyme-free reagent developed for passaging human pluripotent stem cells (7). Importantly, FReP cells exhibit pluripotency marker expression and triploblastic differentiation capabilities similar to those of ESCs and iPSCs (5). From an efficacy standpoint, we previously demonstrated that human FReP cells formed more bone than human iPSCs in a critical-sized SCID mouse calvarial defect model (7). Moreover, unlike iPSCs, implantation of FReP cells in the kidney capsule of immunodeficient mice did not result in teratoma or any other tumor formation (5).

After successfully using FReP cells to form bone, this current study focused on a more difficult challenge of regenerating skeletal muscle. Skeletal muscle constitutes $40 \%-50 \%$ of human body mass, and its significant loss can result in life-altering disabilities $(8,9)$. However, there are no readily available stem or progenitor cell sources for skeletal muscle repair (10-12). Moreover, the highly vascularized microenvironment of the muscle compartment can 
be especially conducive to tumor formation - indeed, intramuscular implantation is a common route for teratoma formation when testing for cellular pluripotency (13). Thus, using nontumorigenic cells as starting materials is a key safety consideration for skeletal muscle regeneration.

Here, we demonstrate the persistence, engraftment, and myogenicity of FReP cells, without tumorigenesis, in the tibialis anterior (TA) muscles of SCID mice. Moreover, FReP cell implantation into environments known to favor pluripotent cell teratoma formation, such as adult male Fox Chase SCID Beige mouse testes (13), did not result in tumor formation during the 4-month experimental period, which confirms the low tumorigenic potency of FReP cells. Furthermore, the FMOD-mediated reprogramming process itself is associated with marked upregulation of cyclindependent kinase inhibitor 2B (CDKN2B; also known as p15) (5) - an additional feature that helps minimize tumorigenicity - in FReP cells and potentially in other pluripotent/multipotent cells.

\section{Results}

FReP cells exhibit significant myogenic differentiation potential in vitro. In vitro, we used an established 2-stage skeletal myogenic differentiation protocol (5) that was developed for conventional retrovirus-mediated BJ fibroblast-derived iPSCs (BJ-iPSCs) (14) on FReP cells. In addition, human satellite cells were used as a positive control in vitro. The differentiated FReP cells expressed myogenic markers in a fashion similar to that of myogenically induced BJ-iPSCs, including myogenic differentiation 1 (MYOD1), sarcomeric $\alpha$-actinin (ACTN), $\alpha$-sarcomeric actin (ACTA1), myosin, and desmin (DES; Figure 1A). In contrast, unreprogrammed parental BJ fibroblasts and FReP-basal cells failed to undergo myogenic differentiation when subjected to the same myogenic protocol (Figure 1A). Since gene profiling is a very reliable tool for analyzing the expression of a focused panel of genes, the "molecular blueprint" of FReP cell myogenic differentiation was comprehensively studied by a commercially available PCR array that revealed significant upregulation of 84 myogenesis-related genes in the myogenically stimulated FReP cells (Figure 1B and Supplemental Table 1; supplemental material available online with this article; https://doi.org/10.1172/JCI125015DS1). In addition, myogenically differentiated FReP cells exhibited creatine kinase activity similar to that of human satellite cells (Figure 1C), indicating comparable in vitro functional myogenic potential between FReP cells and satellite cells $(15,16)$. Taken together, these data suggest that FReP cells are fully capable of behaving like muscle progenitor cells.

FReP cell implantation leads to skeletal muscle generation in vivo. To validate the myogenic potential of FReP cells in vivo, $5 \times$ $10^{5}$ cells that had not undergone any form of premyogenic stimulation were implanted in the left TA muscles of 2-month-old male SCID mice. All negative controls - PBS vehicle (no cells), BJ fibroblasts, and FReP-basal cells - did not alter the TA muscle mass at 6 weeks after implantation (Figure 2A). Only limited numbers of BJ fibroblasts and FReP-basal cells survived in vivo. Surviving BJ fibroblasts were found on the surface of myofibers, while surviving FReP-basal cells were detected in some myofibers (Figure 2B). Meanwhile, retrovirus-mediated BJ-iPSCs, acting as a positive control, showed differentiation and engraft- ment that directly and significantly boosted muscle mass as evidenced by the spatial colocalization of human markers with the skeletal muscle markers (Figure 2B and Supplemental Figure 1). Excitingly, FReP cell implantation increased muscle mass to an even greater extent than retrovirus-mediated BJ-iPSC implantation (Figure 2A). Meanwhile, a broad spatial overlap of human markers with skeletal muscle markers confirmed the myogenic commitment and engraftment of FReP cells in the SCID mouse TA muscles (Figure 2B and Supplemental Figure 1). Overall, FReP cells exhibited superior skeletal muscle generation in vivo when compared with iPSCs.

FReP cells have less tumorigenic potential than iPSCs. Notably, 2 of 8 animals (25\%) that underwent implantation of retrovirusmediated BJ-iPSCs into their uninjured TA muscles experienced tumor formation with active cell proliferation instead of skeletal muscle generation (Figure 2A and Supplemental Figure 2). Neither FReP-basal nor FReP cell implantation led to tumor formation during skeletal muscle (Figure 2) or bone $(5,7)$ regeneration, suggesting less tumorigenic potential than iPSCs.

Since iPSC tumorigenesis is considered to be driven by mutations associated with uncontrollable proliferation $(17,18)$, cellular proliferation was examined. In agreement with previous studies $(5,6)$, retrovirus-mediated BJ-iPSCs exhibited extremely rapid proliferation, while FReP cells proliferated minimally under undifferentiated conditions in vitro (Figure 3A). Next, a soft agar colony formation assay, the standard tumorigenicity test, was used to examine anchorage-independent cellular survivability under a low-nutrient and -oxygen microenvironment (19). After 14 days of cultivation with $10 \mu \mathrm{M}$ Y-27632, the survival of BJ fibroblasts was negligible, while retrovirus-mediated BJiPSCs actively proliferated and formed colonies (Figure 3, B and C). Neither FReP-basal nor FReP cells proliferated or formed colonies; however, FReP-basal cells largely adopted a spindle shape while FReP cells remained morphologically round in the soft agar (Figure 3, B and C).

It is worth noting that the correlation between the soft agar colony formation assay and in vivo tumorigenicity tests is imprecise (20). The soft agar colony formation assay may underestimate the tumorigenic potential of reprogrammed pluripotent/ multipotent cells, because only a small portion of tumorigenic iPSCs form colonies in the gel, owing to the dissociation-induced apoptosis of potentially tumorigenic iPSCs when conducting the assay $(19,21,22)$. Since the intratesticular stromal cells may produce a milieu that is more supportive for implanted cells than subcutaneous or intramuscular microenvironments (13), $1 \times 10^{6}$ cells with $30 \mu \mathrm{L}$ of Matrigel carrier were intratesticularly implanted into Fox Chase SCID Beige mice to further assess the tumorigenicity of FReP cells in vivo. In this system (20), intratesticular implantation of retrovirus-mediated BJ-iPSCs resulted in 100\% (10/10) of the implanted animals developing teratomas with progressive growth (Figure 4A and Supplemental Figure 3B). Excitingly, none of the animals implanted with FReP or FReP-basal cells formed teratomas during the entire 4-month experimental period. Meanwhile, no human markers were detected in mouse testes implanted with FReP cells at 4 months after implantation (Figure 4A). A similar lack of human marker expression was also noted after BJ fibroblast or FReP-basal cell implantation in mice at 
A
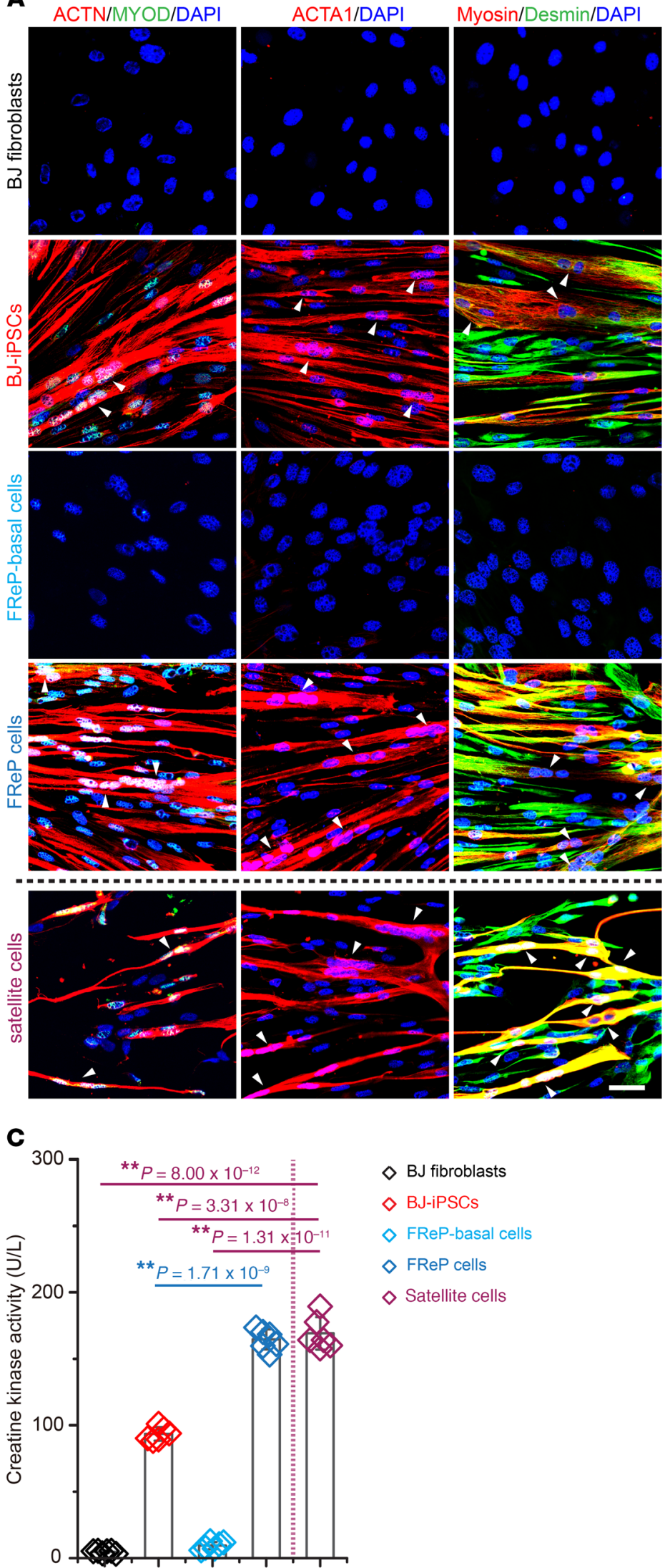

B

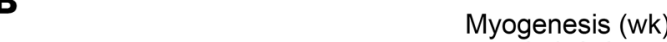

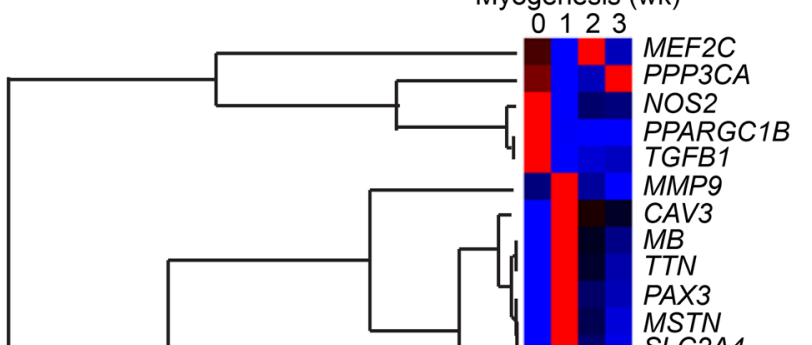

SLC2A4

FBXO32

IGF1

LEP

HK2

PAX7

PDK4

TNF

$A D I P O Q$

ILIB

MYF6

TNNI2

CASP3

MUSK

FOXO1

SGCA

$M Y O D 1$

MYOG

NEB

PRKAG3

TNNT1

MAPK3

MAPK8

LMNA

PRKAB2

CAMK2G

RHOA

ATP2A1

CS

AD

ADRB2

CAPN2

DMPK

AGRN

IGFBP5

CTNNB1

PRKAG1

IKBKB

MAPK 1

FGF2

HDAC5

ACTN3

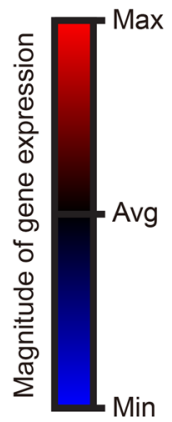

RPS6KB1

$\mathrm{FOXO} 3$

MAPK 14

NFKB1

ACTA1

PRKAA1

$D A G 1$

TNNC1

DMD

MYF5

$D E S$

CAPN3

PPARGC1A

AKT2

AKT2
$D Y S F$

AKT1

MYH2

CAST

CRYAB

IGF2

$M Y H 1$

ACVR2B

$B C L 2$

CAV1

PAARG

BMP4

TNNT3

UTRN

IGFBP3

MYOT 
Figure 1. FReP cells display myogenic differentiation potential in vitro. (A) Myogenic markers ACTN, MYOD, ACTA1, myosin, and desmin were found in FReP cells after cultivation using the established in vitro 2-stage skeletal myogenic differentiation protocol. Under the same differentiation conditions, parental unreprogrammed BJ fibroblasts and FReP-basal cells were used as negative controls, while retrovirus-mediated BJ-iPSCs and differentiated human satellite cells were used as positive controls. White arrowheads indicate the fusing myogenically differentiated cells; scale bar: $100 \mu \mathrm{m}$. (B) A PCR array revealed significantly upregulated myogenesis-related genes in FReP cells during the 3-week in vitro myogenic differentiation period. Unprocessed original Ct data are shown in Supplemental Table 1. $n=3$ independent experiments. (C) The creatine kinase activity assay suggests that, after undergoing myogenic differentiation, $\mathrm{FReP}$ cells have biological activities similar to those of human satellite cells in vitro. ${ }^{* *} P<0.005$ (analyzed by 1-tailed Mann-Whitney and Kruskal-Wallis ANOVA tests); $n=6$.

the same time (Figure 4A). This suggests that, like BJ fibroblasts, FReP-basal and FReP cells that did not undergo predifferentiation stimulation did not spontaneously engraft or differentiate into the host testis tissue. Our previous kidney capsule injection (5) and current intramuscular and intratesticular implantation studies collectively constitute strong evidence that FReP cells are less likely to generate tumors than iPSCs.

FReP cells and iPSCs exhibit different proto-oncogene and tumor suppressor gene expressions. Since FMOD reprogramming is a genome integration-free technology $(5,7)$, the global gene profile of BJ-derived FReP cells (GEO accession number GSE104830) was first compared with that of BJ-derived iPSCs generated through non-genome integration procedures (GEO accession numbers SRR500985/6/7/8, ref. 23; and SRR1583694/5, ref. 24) to gain insight into the mechanisms governing the disparate tumorigenic natures of FReP cells and iPSCs. A total of 2300 highly differentially expressed mRNAs (fold change $\geq 2$ ), identified by the TopHat-Cufflinks package (25) through the Galaxy platform (26), were further analyzed by the DAVID Bioinformatics Resource 6.8 for functional annotation (27). This analysis revealed an enrichment of genes involved in the "pathways in cancer" (Supplemental Figure $4 \mathrm{~A})$ with significant similarity $(\kappa=1.0)$. The differential expression of genes that aligned with human proto-oncogenes and tumor suppressor genes (TSGs) recognized in the UniProt database (28) is displayed graphically (Supplemental Figure 4, B and C).

In comparison with non-genome integration BJ-iPSCs, FReP cells displayed more proto-oncogenes with relatively lower expression levels (Supplemental Figure 4B). Meanwhile, in comparison with retrovirus-mediated BJ-iPSCs used in this study, quantitative reverse transcriptase PCR (qRT-PCR) confirmed that both FReP cells and FReP-basal cells have significantly lower expression levels of proto-oncogenes (Figure 4B) such as MYC, known to markedly accelerate cell proliferation and promote iPSC generation and tumor formation (29), and lin-28 homolog A (LIN28), documented to augment iPSC induction efficiency in a cell division rate-dependent manner (30). Meanwhile, when compared with non-genome integration BJ-iPSCs, FReP cells have a greater number of TSGs with higher expression levels (Supplemental Figure 4C). Further analyses of highly expressed TSGs in FReP cells using the STRING database to retrieve protein-protein interactions (31) emphasized the central roles of tumor protein p53 (TP53 in humans) and CDKN2B (Supplemental Figure 4D).
Accumulating evidence demonstrates that activating TSGs, particularly TP53, cyclin-dependent kinase inhibitor 1A (CDKN1A, also known as $p 21$, which produces a translational product that mediates TP53-induced cell cycle arrest and tumor suppression; ref. 32), $C D K N 2 A$ (also known as p16), and $C D K N 2 B$, reduces the efficiency of iPSC generation and limits the cancerous transformation of iPSCs predominantly by inhibiting cell division (17-19, 30, 33-37). For example, Menendez et al. reported that iPSCs derived from mouse embryonic fibroblasts (MEFs) with an elevated level of p53 (38) or the Ink4a/Arf locus (which encodes Cdkn2B, Cdkn2A, and $A r f$ [an alternative transcript of $C d k n 2 A$ ]) (39) have less tumorigenic potential than iPSCs derived from wild-type MEFs (19). In comparison with parental BJ fibroblasts, retrovirally derived BJ-iPSCs also have significantly less TP53 expression (Figure $5 A)$. As seen in the MEF-derived iPSC study (40), expression of $C D K N 1 A$ and $C D K N 2 B$, but not $C D K N 2 A$, was markedly reduced in retrovirus-mediated BJ-iPSCs (Figure 5, B-D). Meanwhile, these 4 genes were all upregulated in both FReP-basal and FReP cells (Figure 5), which aligns with our previous report in which these 2 populations were not distinguished from one another (5). Notably, higher expression levels of CDKN1A and CDKN2A were observed in FReP-basal cells than in FReP cells (Figure 5, B and $C$ ), while a more robust increase in $C D K N 2 B$ was detected in FReP cells (Figure 5D).

FReP cells reprogrammed from CDKN2B-knockdown fibroblasts acquire teratoma-forming, pluripotent characteristics. To further investigate the effects of TP53 and these CDKNs on the proliferation, multipotency, and tumorigenesis of FReP cells, stable TP53- or CDKN-knockdown (KD) BJ cells were established, respectively, in which the RNAi effects were extended beyond the FMOD reprogramming process (Figure 6A and Supplemental Figure 5). All of the KD BJ cells had reduced CDKN1A levels, while $C D K N 1 A \mathrm{KD}$ did not affect the expression of TP53, $C D K N 2 A$, or $C D K N 2 B$. Also, because of the structure of the INF4A/ $A R P$ locus, expression of $C D K N 2 A$ and $C D K N 2 B$ was decreased by some degree in response to both $C D K N 2 A$ and $C D K N 2 B$ KD (Supplemental Figure 5). The upregulation of $C D K N 2 B$ induced by FMOD reprogramming was also blocked by $C D K N 2 B \mathrm{KD}$ : $C D K N 2 B$ expression increased 21.4-fold in the scrambled shRNAtransfected BJ fibroblasts (scramble FReP cells) in response to FMOD reprogramming; but in $C D K N 2 B-\mathrm{KD} \mathrm{BJ}$ fibroblasts, $C D K N 2 B$ transcription was not altered by continuous FMOD treatment (Figure 6A). Thus, the expression of $C D K N 2 B$ in FReP cells derived from $C D K N 2 B-K D$ BJ fibroblasts $(C D K N 2 B-K D$ FReP cells) was comparable to that seen in BJ-iPSCs.

Although $C D K N 2 B-K D$ FReP cells presented higher expression levels of POU5F1 (also known as OCT4) in comparison with scramble FReP cells or the aforementioned FReP cells derived from BJ fibroblasts without shRNA transfection (in brief, FReP cells; Supplemental Figure 6A), all of these 3 FReP cells had similar multipotent differentiation capabilities and, importantly, myogenic differentiation potentials in vitro (Figure 6, B and C, and Supplemental Figure 6B). To rule out the possibility that the retained multipotent differentiation capability of $C D K N 2 B-K D$ FReP cells was due to inefficient CDKN2B downregulation at the protein level, we carried out Western blotting to further confirm the markedly decreased expression of CDKN2B protein in 
A

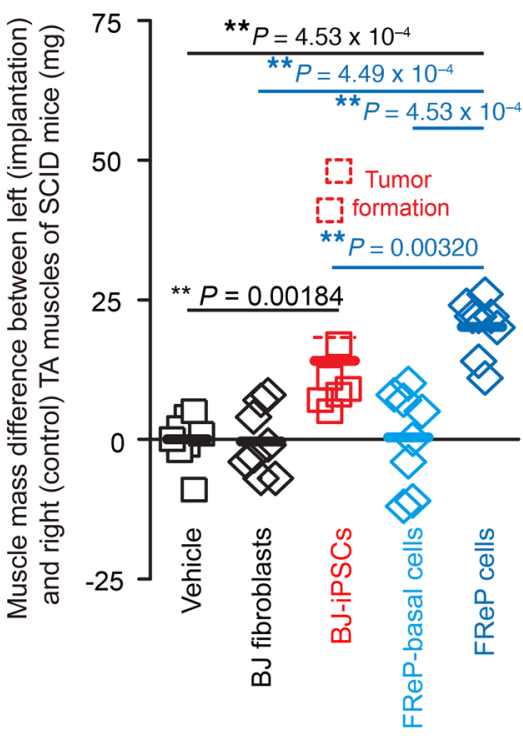

B

Human MHC class I/ Human MHC class I/ Human mitochondria/ ACTA1
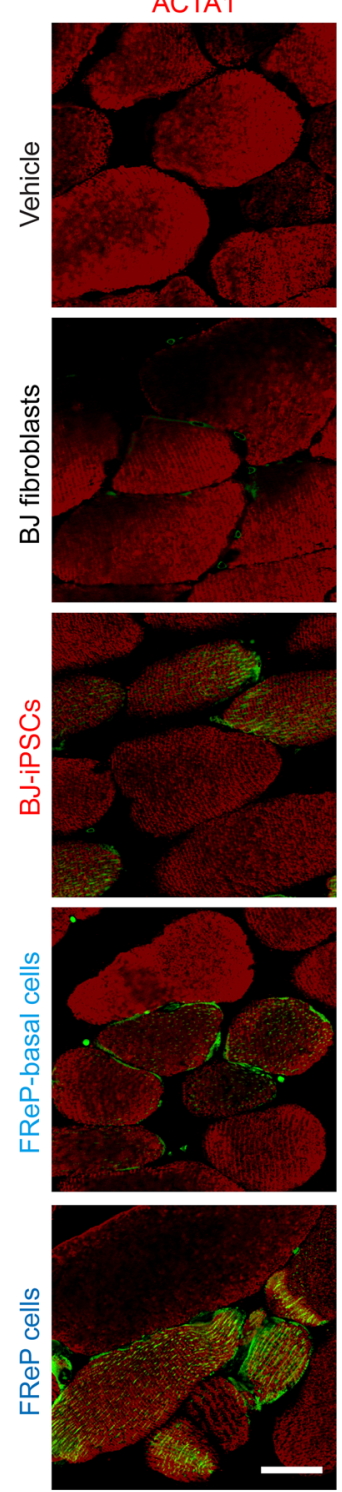
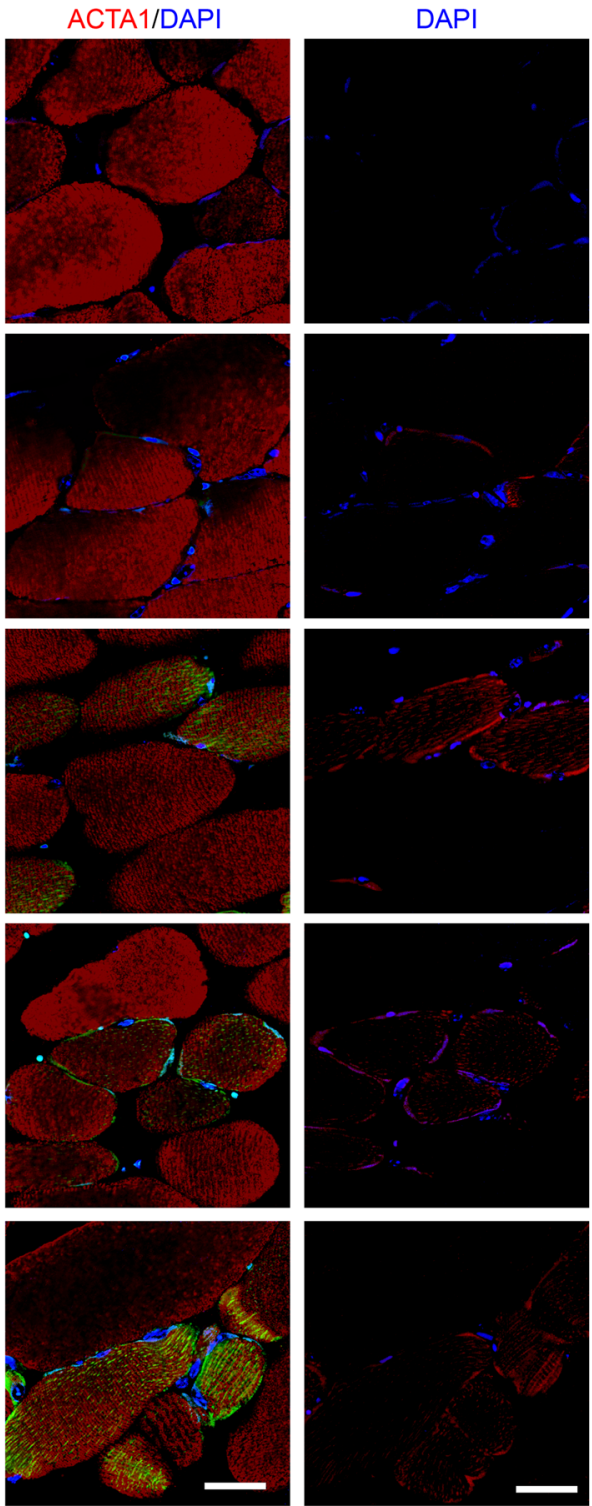
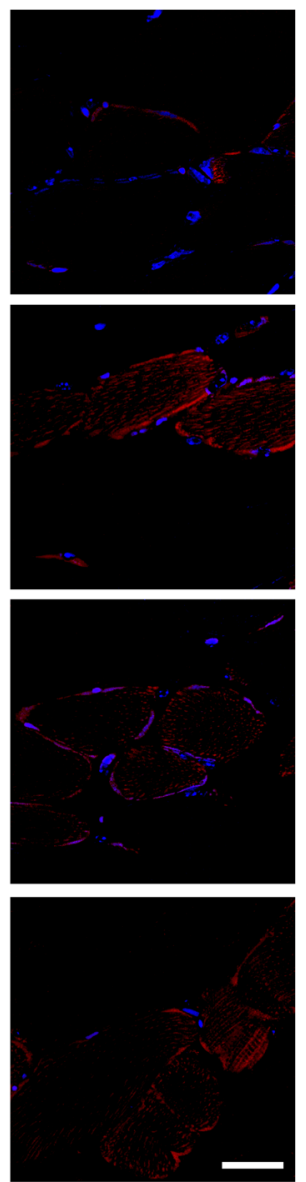

Figure 2. FReP cell implantation in SCID mouse TA muscle leads to the generation of skeletal muscle. (A) TA muscles of SCID mice were weighed, and the left (implantation side) and right (control with no implantation) muscles were compared at 6 weeks after implantation. Two animals implanted with retrovirus-mediated BJ-iPSCs formed tumors (highlighted by dashed lines). Data are presented as mean values. ${ }^{* *} P<0.005$ (analyzed by 1 -tailed Mann-Whitney and Kruskal-Wallis ANOVA tests); $n=8$ or 6 (BJ-iPSC group, excluding the 2 tumor-formation animals whose histological evaluation are shown in Supplemental Figure 2). Black asterisks indicate significance in comparison with the PBS vehicle control group; blue asterisks indicate significance in comparison with the FReP cell-implanted group. (B) Confocal microscopy images showing the coronal section view of SCID mouse TA muscles. Staining of ACTA1 was reduced to better visualize the staining of human MHC class I. The spatial colocalization of skeletal muscle marker ACTA1 with human MHC class I and human mitochondria shows the myogenic differentiation and engraftment of BJ-iPSCs, FReP-basal cells, and FReP cells in vivo. Scale bars: $25 \mu \mathrm{m}$. Confocal microscopy images showing the transverse section view of SCID mouse TA muscles are presented in Supplemental Figure 1.

CDKN2B-KD FReP cells (Supplemental Figure 7). This showed that decreased CDKN2B expression does not diminish the multipotency of FReP cells. Conversely, in agreement with a previous report affirming that permanent p53-p21 pathway suppression impairs the maintenance of iPSC identity (36), TP53- and CDKN1A-KD FReP cells exhibited reduced expressions of all the pluripotent markers, except for NANOG (Supplemental Figure 6A), and impaired myogenic differentiation (Figure 6B) when compared with scramble FReP cells and CDKN2B-KD FReP cells. Surprisingly, TP53, CDKN1A, and $C D K N 2 A \mathrm{KD}$ reduced the multilineage differentiation capacity of FReP cells (Supplemental Figure 6B), suggesting that the upregulation of TP53 and CDKNs is required for FMOD reprogramming and endows FReP cells with their unique triploblastic differentiation potency.

Meanwhile, all tested TP53- or CDKN-KD FReP cells exhibited increased proliferation rates in comparison with FReP cells or scramble FReP cells (Figure 7A). However, in vitro assays demonstrated that only $C D K N 2 B-K D$ FReP cells were able to form colonies in the soft agar, similar to observations in retrovirus-mediated BJ-iPSCs (Figure 7, B and C). Moreover, in comparison with FReP cells and scramble FReP cells, CDKN2B-KD FReP cells had significantly elevated expressions of the aforementioned proto-oncogenes, except for $D E K$ (Figure 7D). Intratesticular implantation of CDKN2BKD FReP cells, but not the other aforementioned knockdown FReP cells, resulted in teratoma formation in 100\% (10/10) of the tested animals (Figure 8A and Supplemental Figure 8E). The CDKN2B-KD FReP cell-derived teratomas were indistinguishable from retrovirus-mediated BJ-iPSC-derived teratomas (Figure $8 \mathrm{~B}$ ). These data constitute the evidence that CDKN2B KD alone converted nontumorigenic, multipotent FReP cells to teratoma-forming pluripotent stem cells.

\section{Discussion}

To address the shortage of tissue-specific progenitor cells, multipotent and pluripotent cells offer exciting alternatives for cell-based tissue repair (4). However, safety concerns, 
A

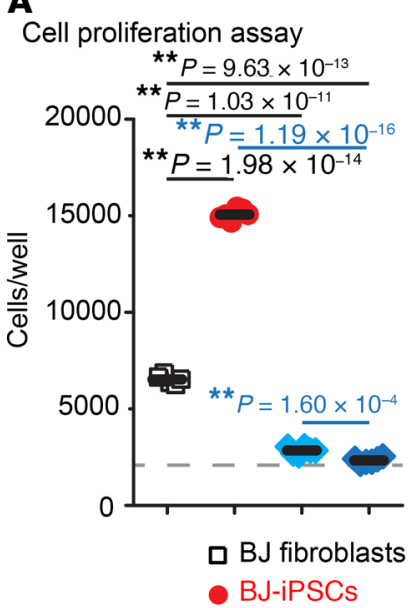

C Soft agar assay
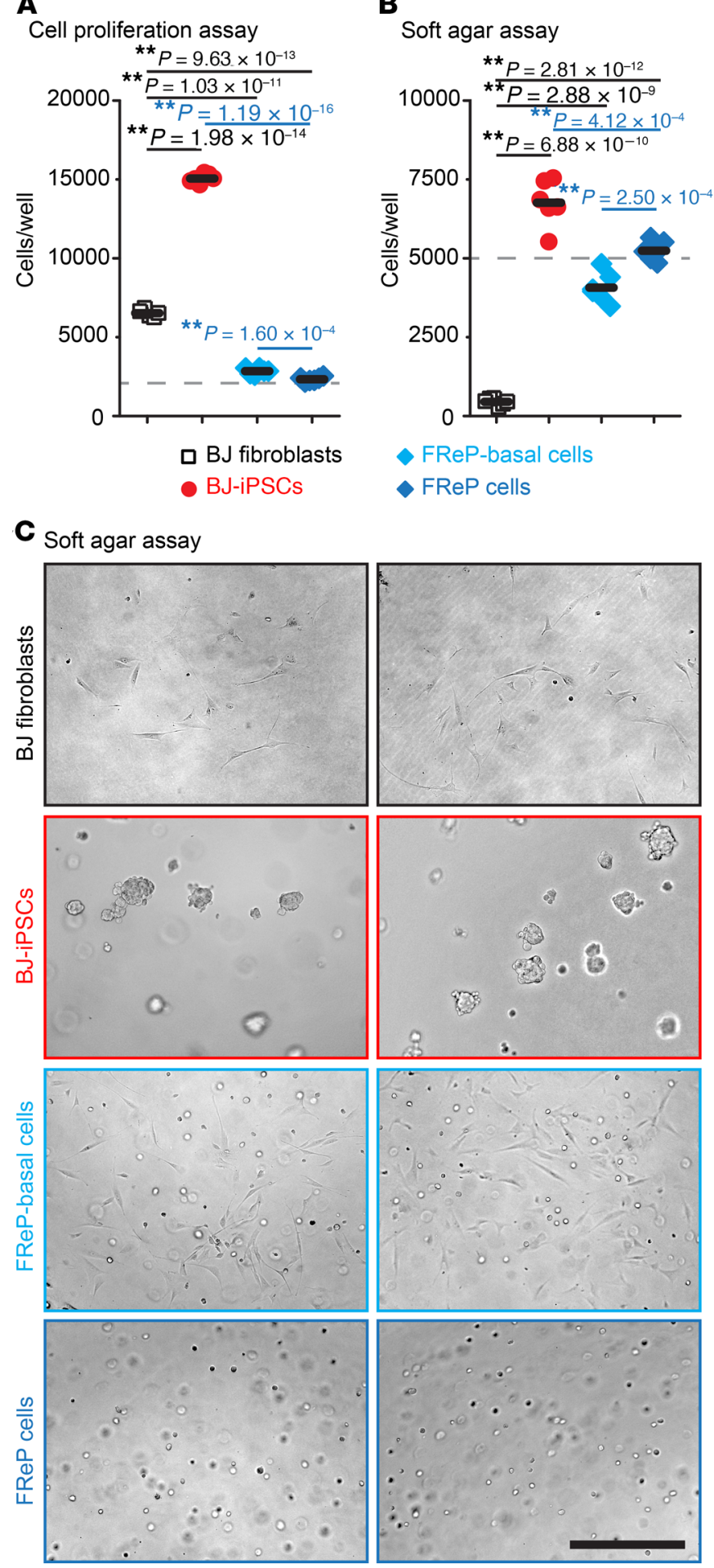

particularly tumorigenicity, must be thoroughly surmounted before the widespread usage of multipotent and pluripotent cells in regenerative medicine (41).

Within the last few decades, MSCs have been widely investigated for their potential use in tissue regeneration because of their multipotent and immunomodulatory properties (42), even though they are generally harvested through more painful and invasive procedures than simple skin biopsy, such as muscle tissue biopsy
Figure 3. FReP cells proliferate minimally in undifferentiating conditions and do not form colonies in a soft agar colony formation assay. (A) A cell proliferation assay was carried out in 96-well culture plates after 2000 cells per well were cultured in undifferentiating conditions for 3 days. (B and C) A soft agar colony formation assay was performed after 14 days of cultivation. Five thousand cells per well were initially seeded. Data are presented as mean values. Gray dashed lines indicate the original cell seeding densities; black asterisks indicate significance in comparison with BJ fibroblasts; blue asterisks indicate significance in comparison with FReP cells. ${ }^{* *} P<0.005$ (A and B; analyzed by 1-way ANOVA and 1-tailed 2-sample $t$ tests); $n=6$; scale bar: $500 \mu \mathrm{m}$ (C).

(43), bone marrow aspiration (44), and liposuction (45). In addition, it is now well documented that MSCs exhibit transient and surprisingly low (less than 1\%) engraftment in newly formed tissues (46, 47). Thus, MSCs assert their regenerative potency by secreting trophic factors instead of directly differentiating into the target tissues (48-52). As an off-target effect, various cytokines, chemokines, and growth factors secreted by MSCs are known to increase the proliferation, migration, and angiogenesis of tumor cells (2). Human MSCs can develop chromosomal aberrations during cultivation (53), undergo spontaneous tumorigenic transformation (54), promote the growth of cocultured glioblastoma cells in vitro, and support glioblastoma development in vivo (3). Animal studies also demonstrate the direct and/or indirect involvement of MSCs in sarcomagenesis (2), especially with inflammatory stimulation (55) that typically arises in injury scenarios. Thus, tumorigenesis is recognized as another risk of using MSCs in humans.

The breakthrough discovery of iPSCs created enthusiasm to use these pluripotent cells for tissue regeneration. However, the widely accepted procedure for iPSC generation, in which transcription factors essential for embryonic development (such as the Yamanaka or Thomson factors) are introduced into the genome of target somatic cells, is known to induce unwanted gene activation and genomic alterations that pose considerable hazards for clinical use (56). For example, in the first clinical trial using iPSC-derived retinal pigment epithelial (RPE) cells to replace age-related macular degeneration RPE cells, 3 DNA deletions were detected in the autologous iPSCs and their derived RPE cells in 1 of the 2 patients (50\%), which halted the planned transplantations (57). Moreover, retrovirus-mediated and transgene-free human iPSCs exhibit similar tumorigenicity with no appreciable difference in teratoma formation capability or teratoma microvascular density $(17,37)$. Although profound efforts are devoted to replacing transcription factors tightly associated with tumor progression with defined chemicals, current research demonstrated that BrdU, a mutation inducer that can incorporate into newly synthesized DNA by replacing thymidine during DNA replication, is absolutely required for achieving chemical induction of pluripotency (58). Considering that iPSCs also possess a potential risk for somatic tumor development that is not present with ESCs (17), the tumorigenic risk of iPSCs remains a credible concern regarding their use clinically.

It has been hypothesized that the pluripotent cell microenvironment plays important roles determining cell fate, as well as the maintenance and induction of pluripotency/multipotency (59). In support of this, early pioneering studies demonstrated the success- 
A

A $\quad$ BJ fibroblasts
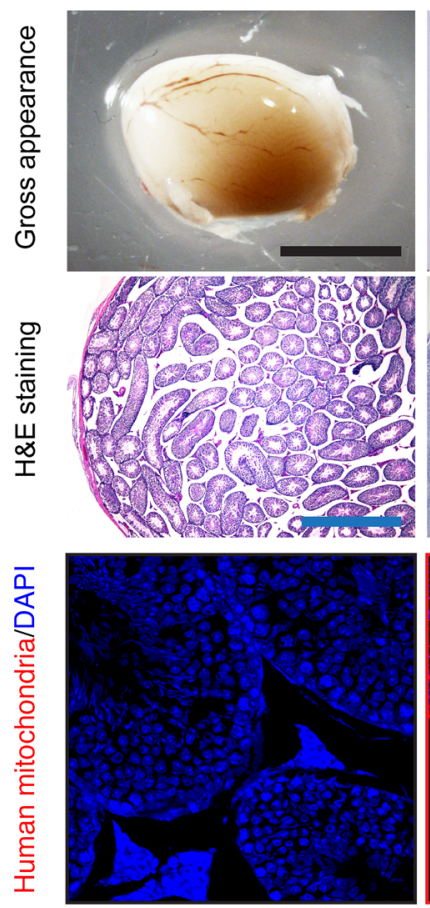

B
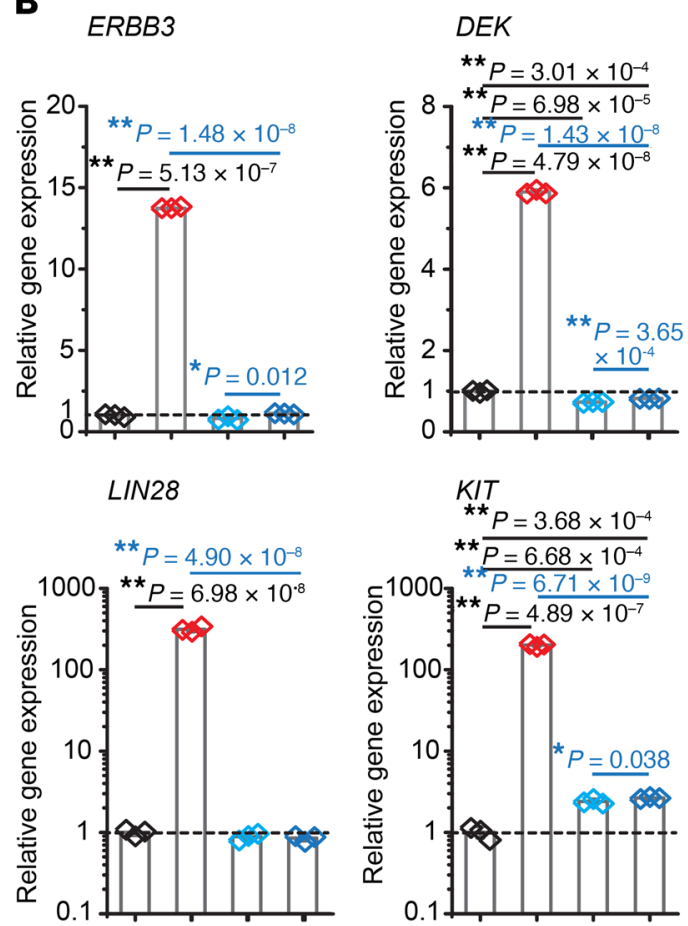

BJ-iPSCs
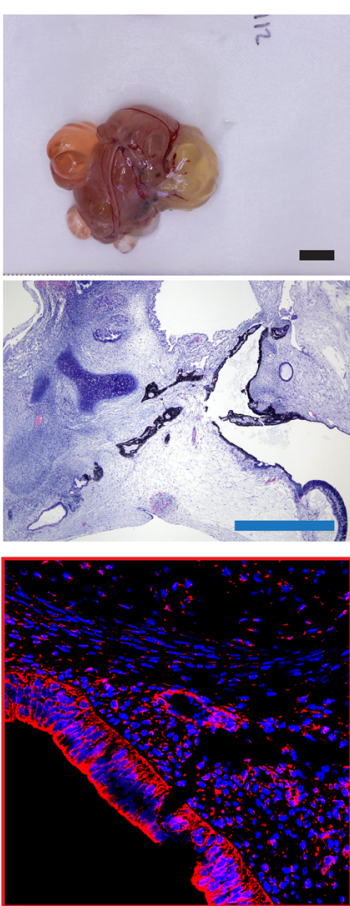

FReP-basal cells
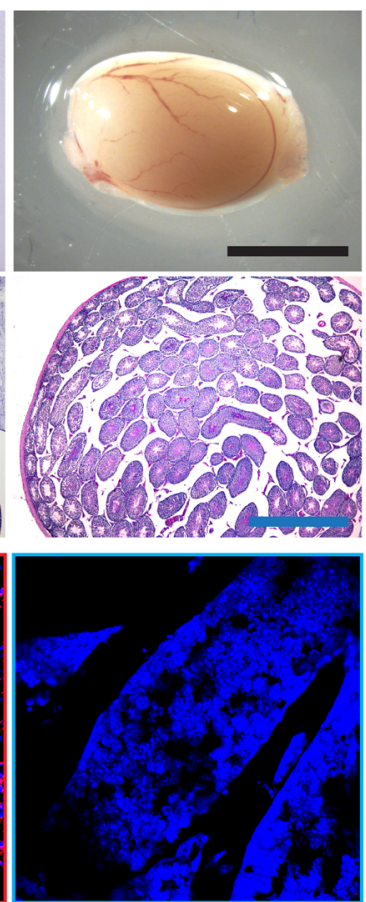

FReP cells
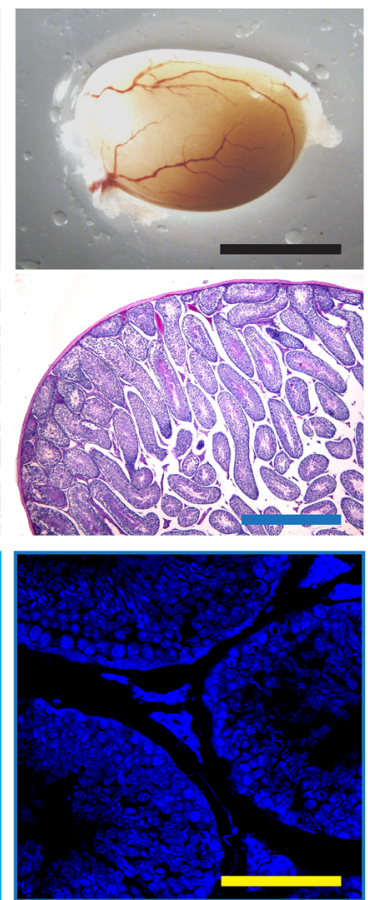

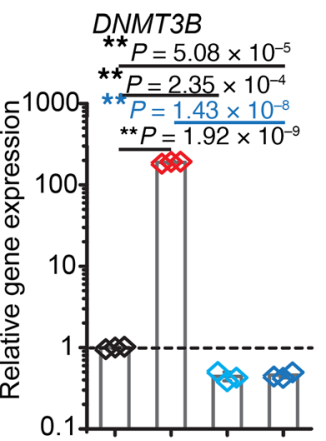

MYC

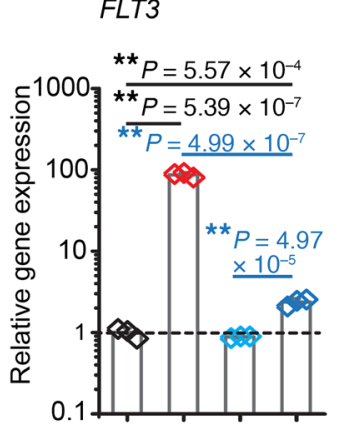

POU2F1
FOX01

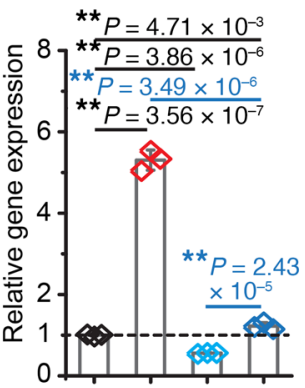

TDGF1
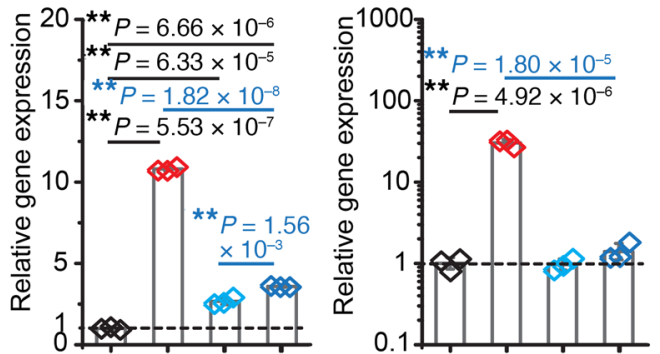

$\diamond$ BJ fibroblasts

$\diamond \mathrm{BJ}$-iPSCs

$\diamond$ FReP-basal cells

$\diamond \mathrm{FReP}$ cells

Figure 4. Intratesticular implantation of FReP cells does not lead to tumorigenesis in Fox Chase SCID Beige mouse testes. (A) Gross appearance and histological evaluation (H\&E staining) of adult Fox Chase SCID Beige mouse testes that were intratesticularly implanted with $1 \times 10^{6}$ cells were documented at 4 months after implantation. All implanted mice testes are shown in Supplemental Figure 3 (10 mice per group). In addition, by tracking of human mitochondria in vivo, significant survival of the implanted human cells was only observed in the BJ-iPSC group, in which teratoma formation was also detected. Scale bars: 5 mm (black), $1 \mathrm{~mm}$ (blue), or $100 \mu \mathrm{m}$ (yellow). (B) The expressions of multiple proto-oncogenes were compared between parental BJ fibroblasts, retrovirus-mediated BJ-iPSCs, FReP-basal cells, and FReP cells. ERBB3, erb-b2 receptor tyrosine kinase 3; DEK, DEK proto-oncogene; DNMT3B, DNA methyltransferase 3B; FLT3, fms-related tyrosine kinase 3; FOXO1, forkhead box 1; LIN28, lin-28 homolog A; KIT, KIT proto-oncogene receptor tyrosine kinase; POU2F1, POU class 2 homeobox 1; TDGF1, teratocarcinoma-derived growth factor 1 . Data are normalized to those of the B) fibroblasts and presented as mean \pm SD. ${ }^{* *} P<0.005$ (analyzed by 1-way ANOVA and 1-tailed 2-sample $t$ tests); $n=3$ independent experiments performed in duplicate. Dashed lines indicate the gene expression levels of BJ fibroblasts; black asterisks indicate significance in comparison with BJ fibroblasts; blue asterisks indicate significance in comparison with FReP cells. 
A

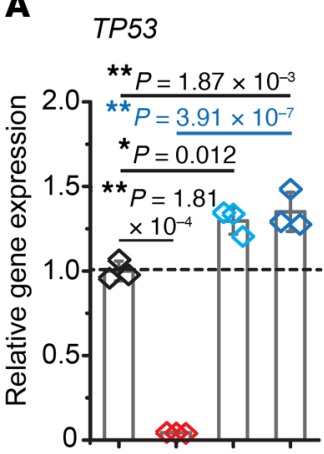

C

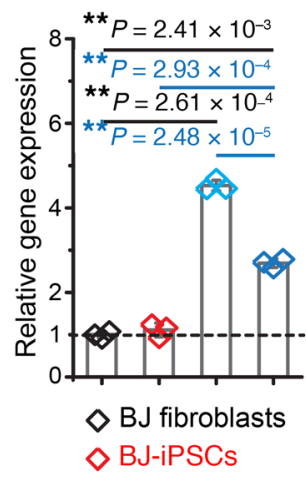

Figure 5. FReP cells have higher expression levels of TP53 and CDKN genes in vitro. Gene expression levels of TP53 (A), CDKN1A (B), CDKN2A (C), and CDKN2B (D) in vitro. Data are normalized to BJ fibroblasts and presented as mean $\pm \mathrm{SD}$. ${ }^{*} P<0.05,{ }^{* *} P<0.005$ (analyzed by 1-way ANOVA and 1-tailed 2-sample $t$ tests); $n=3$ independent experiments performed in duplicate. Dashed lines indicate the gene expression levels of BJ fibroblasts; black asterisks indicate significance in comparison with BJ fibroblasts; blue asterisks indicate significance in comparison with $\mathrm{FReP}$ cells.

ful induction of multipotent stem cells from somatic cells using extracts from Xenopus eggs (60), fish oocytes (61), ESCs (62), and even carcinoma cells (62). Interestingly, recent studies show that fetal MSCs, such as umbilical cord-derived MSCs (UCMSCs), express both specific ESC and adult MSC markers, suggesting that UCMSCs may represent an intermediate cell stage that is developmentally between the adult MSCs and ESCs (63-65). It is also of interest that UCMSCs are predominantly harvested from Wharton's jelly, a proteoglycan-rich connective tissue $(66,67)$; this suggests that recreating a proteoglycan-rich microenvironment may facilitate or induce somatic cell reprogramming to some degree of multipotency/pluripotency. Coincidentally, our previous studies demonstrated that, unlike other small leucine-rich proteoglycan members, FMOD expression markedly decreases from earlygestational fetal skin (which holds the capability to heal scarlessly with restoration of normal dermal extracellular matrix architecture and appendages) to late-gestational fetal and adult skin (in which injury generally results in scar formation; refs. 68, 69). Meanwhile, FMOD loss of function caused scarring in normally scarless earlygestational fetal wounds (69), while FMOD gain of function restored scarless healing to normally scar-prone late-gestational fetal wounds (69) as well as reduced adult scar formation by elicit-

ing a fetal-like phenotype of adult dermal fibroblasts (70). FMOD is also a critical component for the maintenance of endogenous stem cell niches $(71,72)$. These collective data suggest that FMOD is essential for establishing and/or maintaining the fetal and stem cell milieus. To test this hypothesis, we cultured human newborn foreskin BJ fibroblasts and normal adult dermal fibroblasts in FMOD and were able to induce successful reprogramming of these cell populations (5). Excitingly, our previous (7) and current studies demonstrate that the FMOD-reprogrammed FReP cells exhibit a superior capability for bone and skeletal muscle regeneration in comparison with iPSCs. More importantly, the implantation of FReP cells, even in an undifferentiated state, does not cause teratoma or any other kind of tumor formation in vivo in all tests to date. Therefore, FReP cells are a readily available and potentially safer source for musculoskeletal system regeneration as shown here and potentially other regenerative medicine applications.

The decreased tumorigenic potential and proliferation activity, as well as the favorable oncogene and TSG expression profile, also highlight that both the FMOD reprogramming process and the resultant FReP cells are fundamentally distinct from iPSCs. Interestingly, FReP cells bear several critical characteristics of multilineage-differentiating stress-enduring (MUSE) cells $(5,73)$ : they (a) express pluripotent markers, albeit at relatively lower levels than ESCs and iPSCs; (b) can differentiate into all 3 germline cells under specific induction conditions; (c) have low levels of proto-oncogenes, such as LIN28; (d) retain a stable karyotype; and, most importantly, (e) do not form teratomas. Although FReP cells and MUSE cells are both excluded from being considered pluripotent by the stringent mandatory criteria of teratoma formation in vivo, they may represent a different group of cells with a triploblastic differentiation capability that holds tremendous potential in regenerative medicine.

However, FReP cell generation is distinct from MUSE cell collection. Activation and isolation of MUSE cells require severe cellular stress conditions, such as lengthy incubation and digestion, hypoxia, and low temperatures (73), which assist in killing all other viable cells. Conversely, FMOD reprogramming does not require hypoxia or low temperatures, and the resultant FReP cells and FReP-basal cells are both viable. Given the fact that FReP cells resemble quiescent stem cells in multiple ways (5), continuous FMOD exposure may reconstitute a specific microenvironment similar to the niche of quiescent stem cells, and thus induce reprogramming of somatic cells.

Reprogramming and tumorigenesis appear interconnected in many reports examining induced pluripotency (18), although the mechanism governing the transition from nontumorigenic somatic cells to tumorigenic iPSCs remains an enigma. Nevertheless, our current studies demonstrate that FReP cells derived from $C D K N 2 B-K D$ fibroblasts acquired undesirable tumorigenic characteristics. Still, to our knowledge, this represents the first report of conversion of nontumorigenic, multipotent cells obtained via a non-genome integration procedure into a teratoma-forming, pluripotent state simply by suppression of the upregulation of $C D K N 2 B$. These results indicate that, in addition to the conventional transcriptional factor-based procedure, continuous stimulation by FMOD in serum-free conditions while blocking the increase in $C D K N 2 B$ may be an alternative 
A

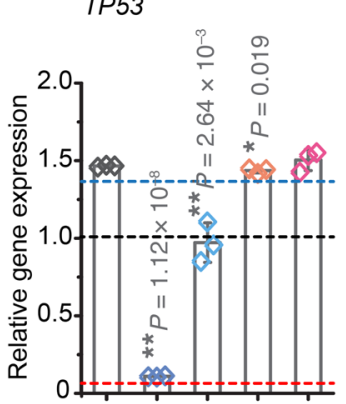

CDKN1A

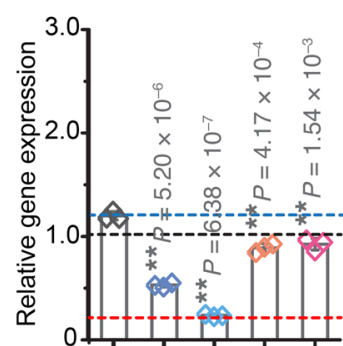

$C D K N 2 A$

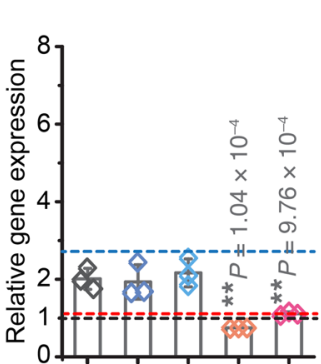

$C D K N 2 B$

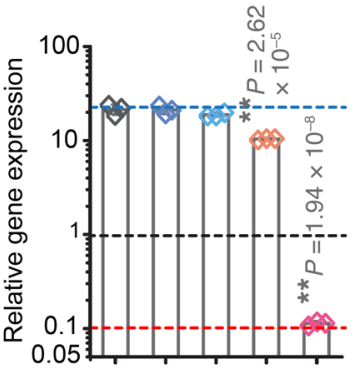

$s \diamond C D K N 2 B-K D$ FReP cells
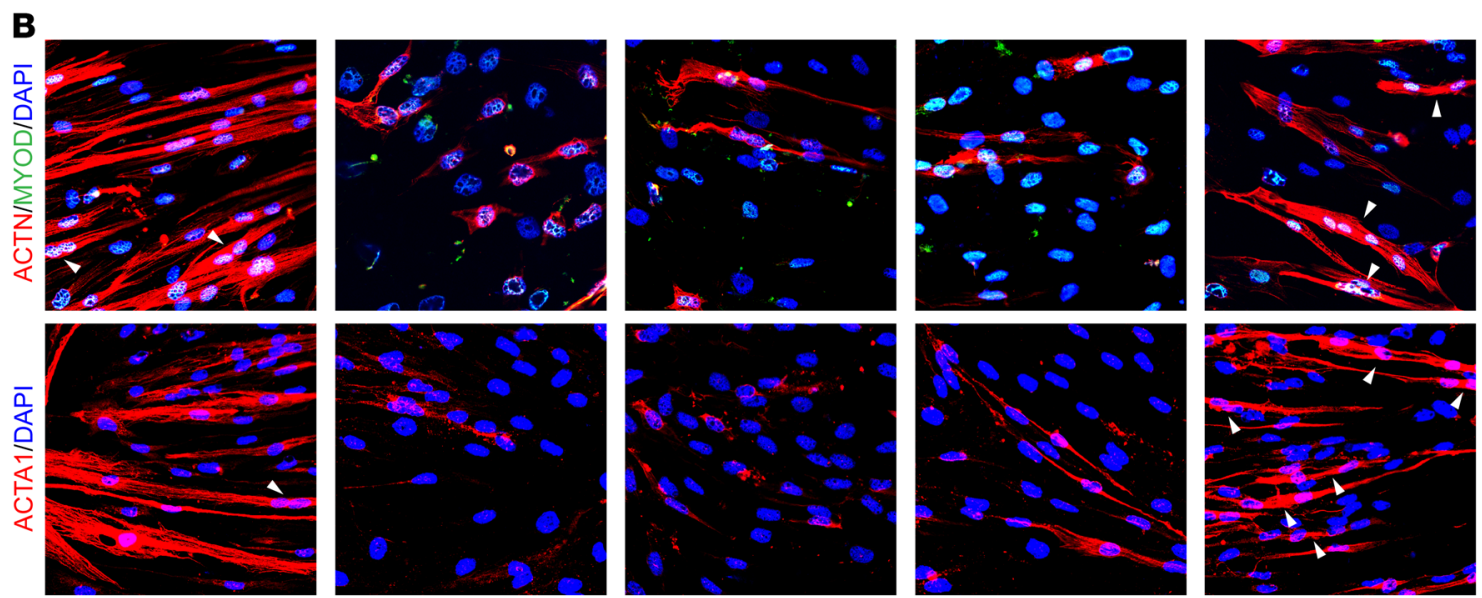

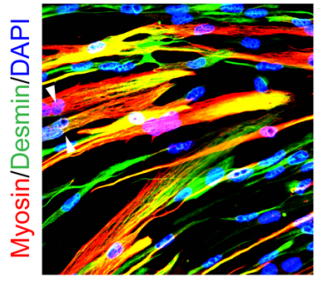

Scramble FReP cells

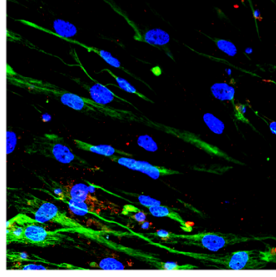

TP53-KD FReP cells

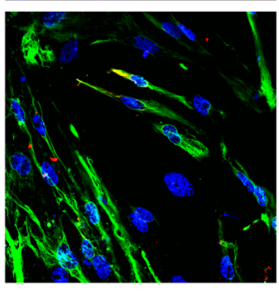

CDKN1A-KD FReP cells
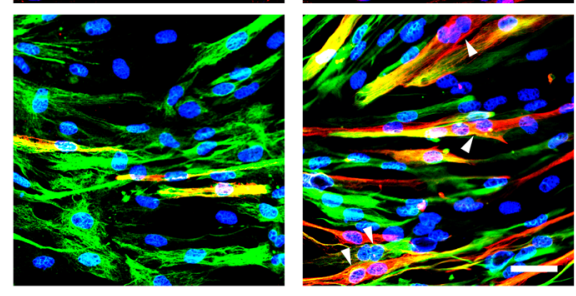

C

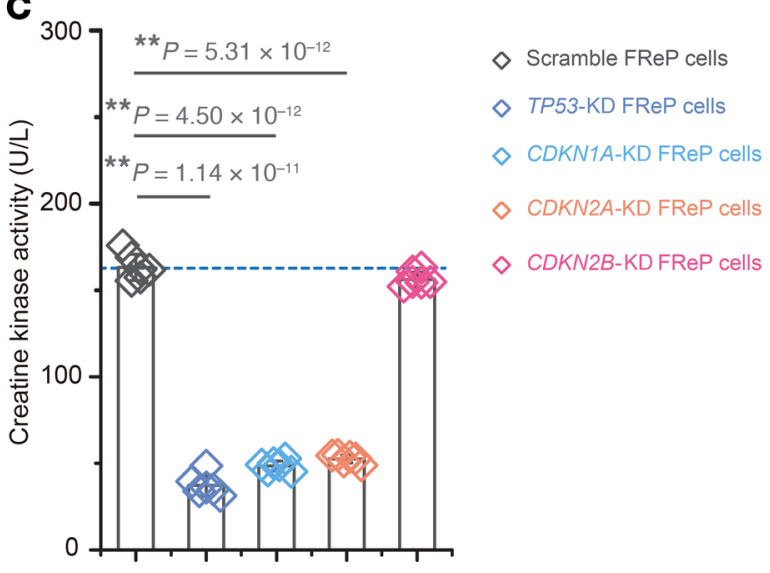

Figure 6. TP53 and CDKN gene-KD FReP cells have different gene expression profiles and myogenic differentiation potentials. (A) Gene expression of TP53 and $C D K N$ genes was assessed in the FReP cells derived from different KD BJ fibroblasts. Data are presented as mean $\pm S D$ and normalized to those of the $B$ J fibroblasts without any shRNA transfection. ${ }^{*} P<0.05$, ${ }^{*} P<0.005$ (analyzed by 1-way ANOVA and 1-tailed 2-sample $t$ tests); $n=3$ independent experiments performed in duplicate. Black dashed lines indicate the gene expression levels of BJ fibroblasts without any shRNA transfection (in brief, BJ fibroblasts); red dashed lines indicate the gene expression levels of BJ-iPSCs; blue dashed lines indicate the gene expression levels of FReP cells derived from BJ fibroblasts without any shRNA transfection (in brief, FReP cells); gray asterisks indicate significance in comparison with FReP cells generated from scrambled shRNA-transfected BJ fibroblasts (scramble FReP cells). (B and C) Myogenic differentiation of KD FReP cells was assessed by myogenic marker staining (B) and creatine kinase activity assay (C) in vitro. White arrowheads indicate the fusing myogenic differentiated cells. Scale bar: $100 \mu \mathrm{m}$ (B). ${ }^{*} P<0.005$ (analyzed by 1-tailed Mann-Whitney and Kruskal-Wallis ANOVA tests, $\mathbf{C}) ; n=6$. strategy to convert somatic cells to pluripotent states. These results highlight the essential role of CDKN2B in governing the transition of multipotent to pluripotent cells, especially during reprogramming procedures without genome integration or oncogene activation.
CDKN2B abnormalities have been associated with many forms of carcinogenesis, including leukemias, carcinomas, and melanomas. As an effector of TGF $\beta$, CDKN2B inhibits cyclindependent kinase $4 / 6$, and thus induces a $G_{1}$-phase cell cycle arrest (74). However, since most previous studies focused on 
A

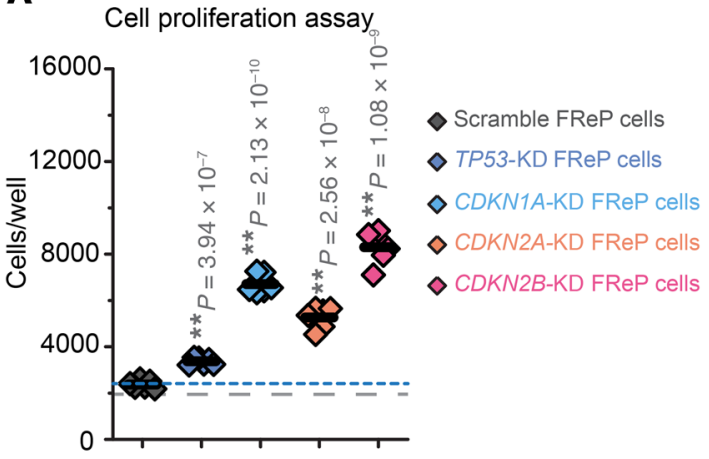

B

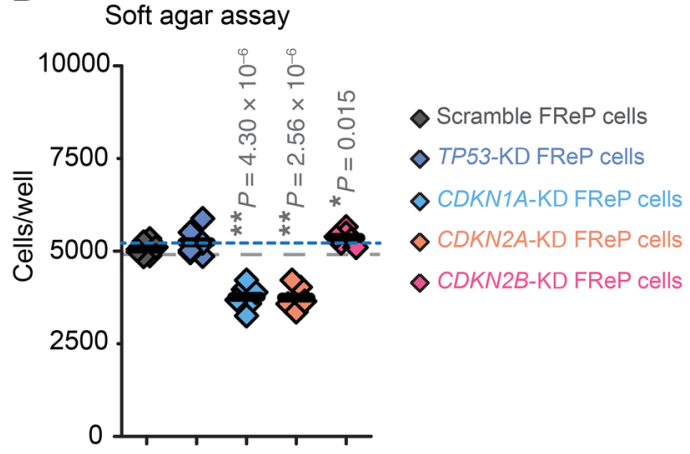

c

Soft agar assay
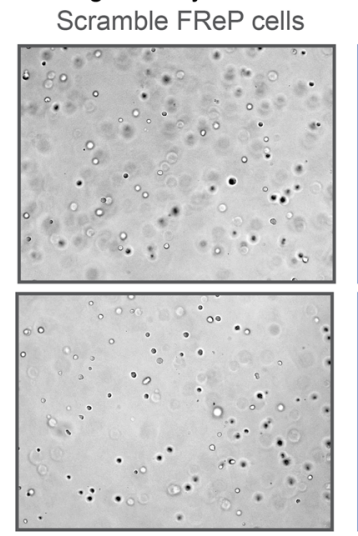

D

ERBB3
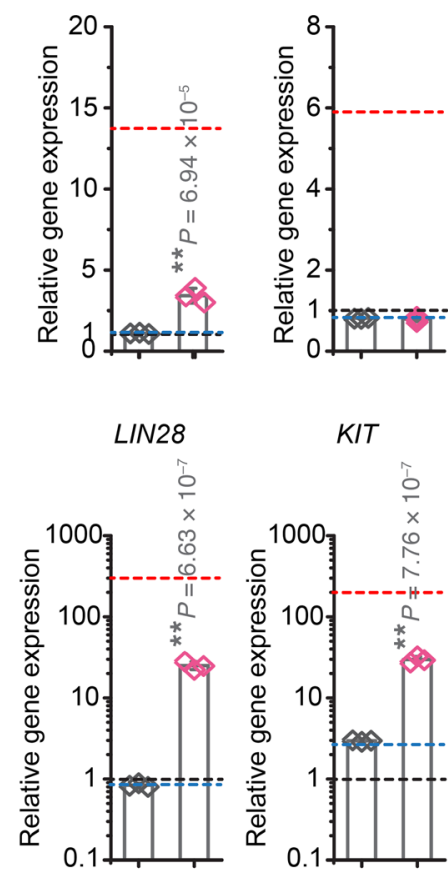

KIT

TP53-KD FReP cells
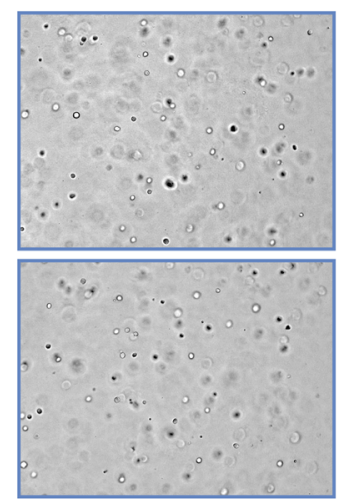

DEK

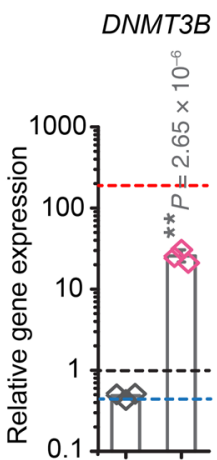

MYC

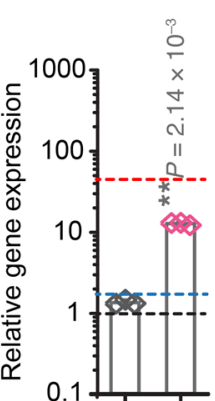

CDKN1A-KD FReP cells
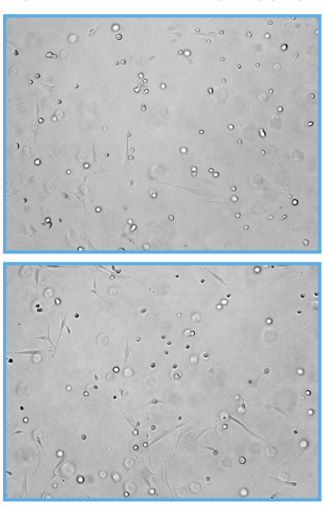

FLT3

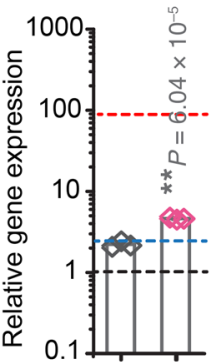

POU2F1

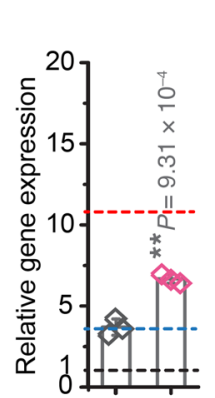

CDKN2A-KD FReP cells
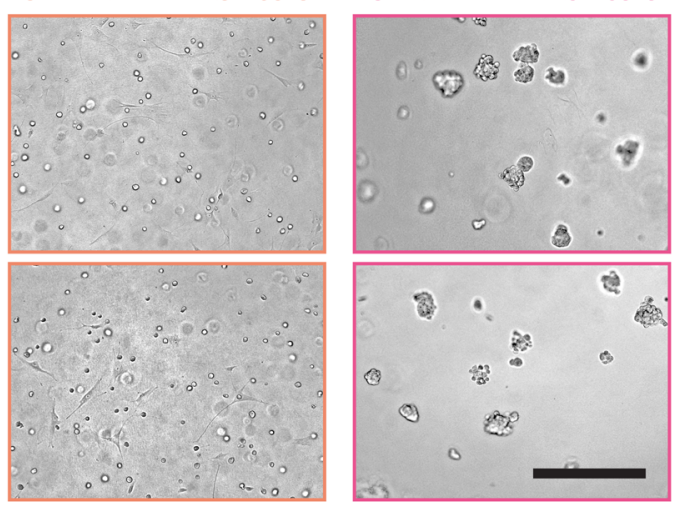

CDKN2B-KD FReP cells
FOX01

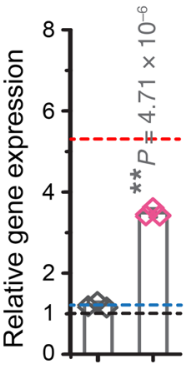

TDGF1

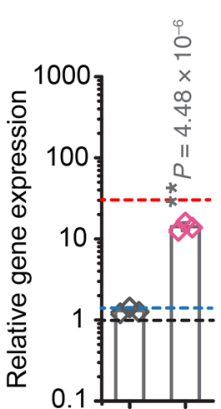

Figure 7. TP53 and CDKN gene KD diversely alters FReP cell proliferation, anchorageindependent survival ability in soft agar, and proto-oncogene expression in vitro. (A) A cell proliferation assay was carried out in 96-well culture plates after 2000 cells per well were cultured in undifferentiating conditions for 3 days. (B and C) A soft agar colony formation assay was performed after 14 days of cultivation. Five thousand cells per well were seeded initially. (D) The expressions of multiple proto-oncogenes in scramble FReP cells and CDKN2B-KD FReP cells were compared. Data are presented as mean values (A and $\mathbf{B}$ ) or mean values normalized with those of the $B$ J fibroblasts \pm SD (D). ${ }^{* *} P<0.005$ (analyzed by 1-way ANOVA and 1-tailed 2-sample $t$ tests); $n=6(\mathbf{A}-\mathbf{C})$ or 3 independent experiments performed in duplicate (D). Gray dashed lines indicate the original cell seeding densities (A and $\mathbf{B}$ ); black dashed lines indicate the gene expression levels of BJ fibroblasts (D); red dashed lines indicate the gene expression levels of BJ-iPSCs (D); blue dashed lines indicate the cell densities ( $\mathbf{A}$ and $\mathbf{B}$ ) or the gene expression levels (D) of FReP cells, respectively; gray asterisks indicate significance in comparison with scramble FReP cells. Scale bar: $500 \mu \mathrm{m}$.

$$
\diamond \begin{aligned}
& \text { Scramble } \\
& \text { FReP cells }
\end{aligned} \quad \diamond \begin{aligned}
& \text { CDKN2B-KD } \\
& \text { FReP cells }
\end{aligned}
$$


the $I N F / A R F$ locus as a whole without separating $C D K N 2 B$ from $C D K N 2 A$, these studies did not fully elucidate the isolated function of CDKN2B. Hence, the contribution of low CDKN2B levels to tumorigenesis may be underestimated. Taking a closer look at the INK4/ARF locus, CDKN2A seems to play a more vigorous role in oncogenesis than CDKN2B. For instance, in acute lymphoblastic leukemia, $C D K N 2 A$ is often deleted without $C D K N 2 B$ deletion, while a sole $C D K N 2 B$ deletion is almost never detected (75). However, our current results demonstrate that sustained suppression of $C D K N 2 B$, instead of $C D K N 2 A$, during FMOD reprogramming plays the predominant role in introducing a tumorigenic potential into the nontumorigenic FReP cells. This suggests that CDKN2A and $\mathrm{CDKN} 2 \mathrm{~B}$ may have different bioactivities and that further targeted studies on the role of CDKN2B in reprogramming, maintenance, and tumorigenesis of multipotent/pluripotent cells, including MUSE cells and iPSCs, are warranted.

Moreover, on the basis of the clear, inverse relationship between tumorigenesis and CDKN2B levels observed in this study, elevating CDKN2B expression may be an interesting, alternative antitumor strategy to decrease the tumorigenic risk of the therapies based on pluripotent/multipotent cells (such as iPSCs). However, it is worth noting that iPSCs exhibit significantly lower INK4/ $A R F$ locus expression than their parental fibroblasts (76). In particular, $C D K N 2 B$ is more severely repressed during iPSC reprogramming than $C D K N 2 A$ and $A R F$ (76). Meanwhile, transient silencing of the INK4/ARF locus is sufficient to accelerate the reprogramming progress and increase the number of iPSCs (76). Since INK4/ $A R F$ locus inhibition promotes iPSC generation, it is highly possible that forcing $C D K N 2 B$ expression may fundamentally impede the reprogramming process that yields iPSCs. An alternative strategy to test whether a CDKN2B-based antitumor strategy would work in iPSCs is to consistently upregulate CDKN2B expression in fully reprogrammed iPSCs to suppress their tumorigenic potency. However, despite the low efficiency, the potential off-target effects of human iPSC genome editing often lead to high cell mortality and, more importantly, introduce mutations that are independent of the gene of interest, and thus confound the analysis of the tumorigenic risk of the genome-edited cells (77-82). Even more crucially, because of the cluster-forming nature of iPSCs, it would be a major challenge to purify $C D K N 2 B$-overexpressing iPSCs from the surrounding iPSCs without CDKN2B upregulation, especially since there is no known surface marker that could assist this process currently. Forcing CDKN2B expression in reprogrammed iPSCs may also not be a practical and reliable way to reduce the tumorigenic risk of iPSCs, as even only a small portion of tumorigenic iPSC contamination can induce teratoma formation (83). Therefore, aside from understanding whether CDKN2B can prevent iPSC tumorigenesis, how to reliably evaluate and effectively implement the CDKN2B overexpression strategy in iPSCs remains a key challenge for future studies.

In summary, we demonstrated that FReP cells exhibit a superior capability for skeletal muscle regeneration with markedly less tumorigenic risk when compared with iPSCs. As FReP cells are fundamentally distinct from iPSCs, FReP cells may represent another class of cells with a triploblastic differentiation capability rather than teratoma-forming pluripotency that may have greater applications in regenerative medicine. Concomitantly, we iden- tified that decreased FReP cell tumorigenicity is directly related to $C D K N 2 B$ upregulation during the FMOD reprogramming process, highlighting an essential role for CDKN2B in regulating cell fate and tumorigenesis.

\section{Methods}

Cell culture. Human newborn foreskin BJ fibroblasts (ATCC CRL-2522; ATCC) were cultured in a 4:1 mixture of DMEM (containing $4 \mathrm{mM}$ L-glutamine, $1.0 \mathrm{~g} / \mathrm{L}$ glucose, and $1.5 \mathrm{~g} / \mathrm{L}$ sodium bicarbonate; Thermo Fisher Scientific) and Medium 199 (Thermo Fisher Scientific), supplemented with 10\% FBS (Thermo Fisher Scientific) and 1\% penicillin/ streptomycin (P/S; Thermo Fisher Scientific) (7). BJ fibroblasts were authenticated by Laragen Inc. and tested negative for mycoplasma contamination using the Universal Mycoplasma Detection Kit (ATCC; Supplemental Figure 9). BJ-iPSCs obtained by conventional retrovirusmediated methods (14) were maintained on plates precoated with Matrigel human ESC-qualified (hESC-qualified) Matrix (BD Biosciences) with mTESR1 medium (STEMCELL Technologies) (7).

Human satellite cells were purchased from Lifeline Cell Technology and cultured according to the manufacturer's instructions. After 1 week of culture in MyoLife Complete Myogenesis Differentiation Medium (Lifeline Cell Technology), the differentiated human satellite cells were used as a positive control for in vitro myogenic differentiation.

FMOD production. cDNA of a human FMOD transcript (GenBank NM_002023) was subcloned into a commercially available vector, pSecTag2A (Thermo Fisher Scientific), with a C-terminal His tag, and then transfected into CHO-K1 cells (ATCC) (84). After establishment of a stable FMOD expression clone, FMOD was produced and purified by a contract research organization, GenScript. Briefly, a stable human recombinant FMOD-expressing CHO-K1 cell line was cultured in $1 \mathrm{~L}$ of serum-free Freestyle CHO Expression Medium (Thermo Fisher Scientific) at $37^{\circ} \mathrm{C}$ with $5 \% \mathrm{CO}_{2}$ in an Erlenmeyer flask. The cell culture supernatant was harvested after 10 days for purification with a HiTrap IMAC HP 1-mL column (GE Healthcare). The fractions from a 100-mM imidazole elution were collected and dialyzed against 20 mM PBS, pH 7.4. Next, the sample with low conductivity was loaded onto a HiTrap Q HP 1-mL column (GE Healthcare) for further purification. FMOD was then purified under nonreducing conditions, dialyzed again (7), and then subjected to lyophilization. The purity of the FMOD product was $85 \%$. FMOD was reconstituted in PBS and then underwent sterilization through a $0.22-\mu \mathrm{m}$ filter (Thermo Fisher Scientific) before use (70).

FMOD reprogramming. BJ fibroblast cells $\left(4 \times 10^{5}\right.$ per well $)$ were seeded in 6-well culture plates overnight to confluence and then exposed to $0.4 \mathrm{mg} / \mathrm{mL}$ recombinant human FMOD in DMEM supplemented with $1 \% \mathrm{P} / \mathrm{S}$ for reprogramming under serum-free conditions. The medium was exchanged with fresh medium daily (5, 7). After 21 days of continuous FMOD treatment, FReP cells were harvested by ReLeSR (an enzyme-free hESC and hiPSC selection and passaging reagent; STEMCELL Technologies), which significantly increased the yield of FReP cells in comparison with a traditional manual scraping method (Supplemental Figure 10). The yielded cells were then cultured on Matrigel hESC-qualified Matrix-coated (BD Biosciences) plates with mTESR1 medium (STEMCELL Technologies) (7).

$R N A i$. Following the manufacturer's instructions, plasmids harboring TP53 shRNA, CDKN1A shRNA, CDKN2A shRNA, or CDKN2B shRNA (Santa Cruz Biotechnology) were used to knock down the 
expression of the respective target genes. Control shRNA Plasmid-A (Santa Cruz Biotechnology), which encoded a scrambled shRNA sequence, was used as a negative control for RNAi. For each target gene, the colony with the lowest gene expression of the target gene was selected from 5 candidate knockdown colonies for further investigation.

Proliferation assay. Two thousand cells per well were seeded into 96-well culture plates for a proliferation assay. After 3 days of incubation, cell proliferation was assessed by the Vybrant MTT Cell Proliferation Assay Kit (Molecular Probes) with an Epoch Microplate Spectrophotometer coupled with Gen5 software (version 2.04.11, BioTek Instruments Inc.).

Soft agar colony formation assay. Soft agar colony formation assay was performed with the CytoSelect 96-Well In Vitro Tumor Sensitivity Assay (Cell Biolabs Inc.) according to the manufacturer's instructions. Five thousand cells per well were initially seeded with the supplied medium and $10 \mu \mathrm{M}$ Rho-associated protein kinase (ROCK) inhibitor Y-27632 (STEMCELL Technologies). After 14 days of cultivation, representative photographs were captured on an Olympus IX71 microscope coupled with a DP73 camera and cellSens Standard 1.9 software (Olympus). Cellular anchorage-independent growth was quantified according to the instructions of the CytoSelect 96-Well In Vitro Tumor Sensitivity Assay with an Epoch Microplate Spectrophotometer.

In vitro 2-stage skeletal myogenic differentiation. Cells were seeded on Attachment Factor Protein-coated plates with myogenic medium I (DMEM supplemented with 10\% FBS, 1\% P/S, 10\% horse serum [HS; Thermo Fisher Scientific], and 1\% chicken embryo extract [CEE; Gemini Bio Products]) for 7 days. Then, myogenic medium II (DMEM supplemented with $1 \% \mathrm{FBS}, 1 \% \mathrm{P} / \mathrm{S}, 1 \% \mathrm{HS}$, and $0.5 \% \mathrm{CEE}$ ) was used for another 14 days of cultivation. Half of the medium was renewed every 4 days $(5,85)$.

Antibodies against ACTA1 (catalog ab28052, clone Alpha Sr-1, Abcam), ACTN (catalog ab9465, clone EA-53, Abcam), desmin (catalog ab8592, Abcam), MYOD (catalog ab64159, Abcam), and myosin (catalog MAB1628, clone NOQ7.5.4D, MilliporeSigma) were used to confirm skeletal myogenic differentiation. All immunostaining, in terms of concentration, antigen retrieval, etc., was conducted based on the instructions of the manufacturers.

One microgram total protein of lysate from cells that underwent myogenic differentiation in vitro was used for a creatine kinase activity test $(15,16)$ using the Creatine Kinase Activity Assay Kit (MilliporeSigma) according to the manufacturer's protocol. The test was performed at $37^{\circ} \mathrm{C}$, and results were documented with an Epoch Microplate Spectrophotometer.

In vitro neurogenic differentiation. Cells were seeded on AggreWell 800 Plates with AggreWell medium (STEMCELL Technologies) for embryoid body (EB) formation, following the manufacturer's instructions $(5,7)$. EBs were then transferred to a 6-well Ultra Low Cluster Plate (Costar, Corning Inc.) with KnockOut DMEM (Thermo Fisher Scientific) supplemented with 10\% KnockOut Serum Replacement (Thermo Fisher Scientific), 2 mM L-glutamate (Thermo Fisher Scientific), $1 \% \mathrm{P} / \mathrm{S}, 10 \mu \mathrm{M}$ all-trans retinoic acid (RA; Santa Cruz Biotechnology), and $100 \mathrm{nM}$ of the N-terminal active fragment of human sonic hedgehog (Shh; R\&D Systems) to generate spheres. Fresh RA was added every day, and the medium and supplements, including Shh, were replaced every 3 days. After 8 days of suspension culture, the induced spheres were transferred onto poly-ornithine/fibronectincoated (MilliporeSigma) plates with DMEM/F12 medium (Thermo
Fisher Scientific) supplemented with 2\% FBS, 1\% P/S, N2 Supplement (Thermo Fisher Scientific), $20 \mathrm{ng} / \mathrm{mL}$ glial-derived neurotrophic factor (Thermo Fisher Scientific), $20 \mathrm{ng} / \mathrm{mL}$ brain-derived neurotrophic factor (Thermo Fisher Scientific), $20 \mathrm{ng} / \mathrm{mL}$ ciliary neurotrophic factor (Thermo Fisher Scientific), and 1× B27 serum-free supplement (Thermo Fisher Scientific) $(5,86,87)$. The medium was changed every 3 days. After 9 days, cells were fixed for immunocytochemistry examination with antibodies against neuron-specific $\beta_{\mathrm{III}}$-tubulin (also known as TUJ1; catalog ab18207, Abcam) $(5,7)$.

In vitro osteogenic differentiation. Cells were seeded on Attachment Factor Protein-coated (Thermo Fisher Scientific) plates and cultured with MEM Alpha (Thermo Fisher Scientific) supplemented with 10\% FBS, $50 \mu \mathrm{g} / \mathrm{mL}$ ascorbic acid (MilliporeSigma), $10 \mathrm{mM} \beta$-glycerophosphate (MilliporeSigma), and 1\% P/S for 28 days. The medium was changed every 3 days. Mineralization was detected by alizarin red staining (LifeLine Cell Technology) $(5,7)$.

In vitro pancreatic differentiation. Cells were cultured in RPMI 1640 medium (Thermo Fisher Scientific) supplemented with 2\% FBS, $2 \mathrm{mM}$ L-glutamate, $1 \% \mathrm{P} / \mathrm{S}$, and $100 \mathrm{ng} / \mathrm{mL}$ recombinant activin A (Thermo Fisher Scientific) for 4 days to differentiate into an endoderm derivate. Cells were cultured for another 16 days without activin A $(5,88,89)$ before characterization with antibodies against Nkx6.1 (catalog F55A10, clone F55A10, Developmental Studies Hybridoma Bank), insulin (catalog 4590, Cell Signaling Technology), pancreatic and duodenal homeobox 1 (PDX1; catalog ab47267, Abcam), and amylin (catalog GTX11022, clone R10/99, GeneTex).

Immunocytochemistry. Samples selected for immunocytochemistry staining were fixed in prechilled acetone. DAPI (MilliporeSigma) was used for nuclear staining $(5,7)$. The StemLite Pluripotency Kit (catalog 9656, Cell Signaling Technology) was used to detect a panel of proteins that are specifically expressed in human pluripotent cells (5). Images were collected with the Leica TCS SP8 Confocal Laser Scanning Platform (Leica).

RNA isolation. RNA was extracted using the RNeasy Mini Kit (Qiagen) with DNase (Qiagen) treatment to ensure that the samples were not contaminated with genomic DNA. RNA purity was assessed by the Epoch Microplate Spectrophotometer.

PCR array quantitative RT-PCR. PCR arrays are one of the most reliable tools for analyzing the expression of a focused panel of genes. To further establish the feasibility of FReP cells as an alternative safe cell source for skeletal muscle regeneration, we used PCR arrays to generate a "molecular blueprint" of FReP cell activities during myogenic differentiation. According to the manufacturer's instruction, the $\mathrm{RT}^{2}$ Profiler PCR Array for Human Skeletal Muscle (Qiagen) was used to track the expression of myogenic-related genes. Three different cDNA templates were tested.

$q R T-P C R$. For qRT-PCR, $1.0 \mu \mathrm{g}$ RNA was used for a reverse transcriptase reaction with iScript Reverse Transcription Supermix for RT-qPCR (Bio-Rad). One microliter product was used for real-time PCR with SsoAdvanced Universal Probes Supermix (Bio-Rad) and TaqMan primers/probe sets (Thermo Fisher Scientific) on a QuantStudio3 system (Thermo Fisher Scientific). Three different cDNA templates were tested in duplicate $(5,7)$.

Whole transcriptome sequencing. Whole transcriptome sequencing (RNA-Seq) of FReP cells was performed by the UCLA Technology Center for Genomics \& Bioinformatics. Briefly, Illumina (HiSeq 2500, Illumina) $1 \times 50 \mathrm{bp}$ short reads were aligned to the transcriptome 
A

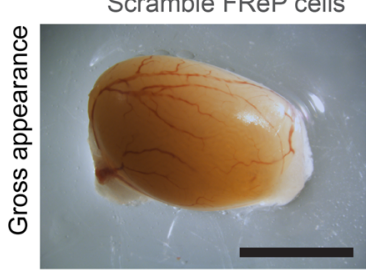

TP53-KD FReP cells

CDKN1A-KD FReP cells

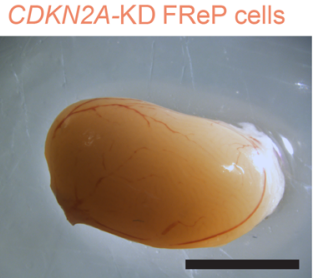

CDKN2B-KD FReP cells
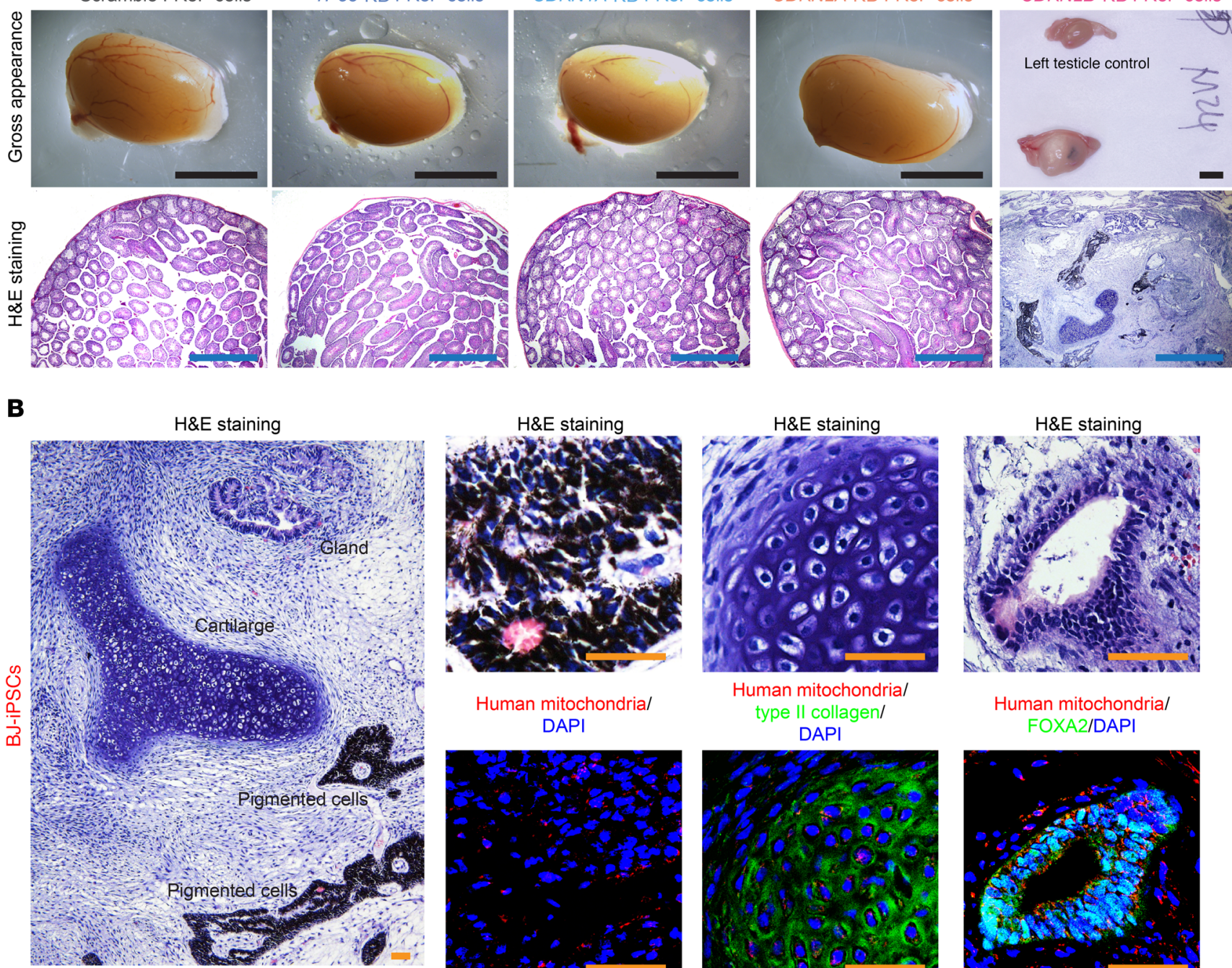

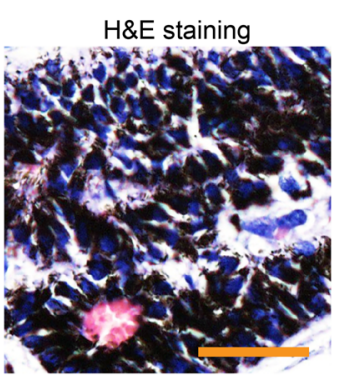

Human mitochondria/

DAPI

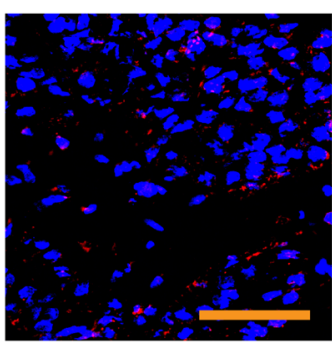

H\&E staining

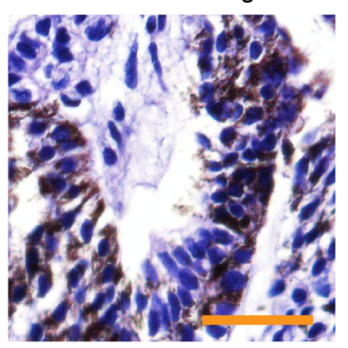

Human mitochondria/

DAPI

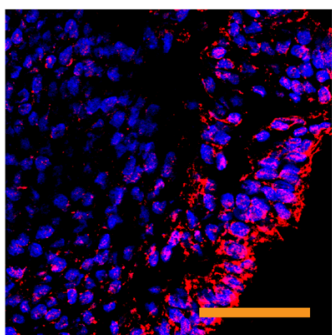

Ectoderm

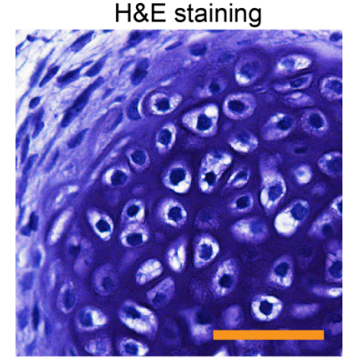

Human mitochondria/

DAPI

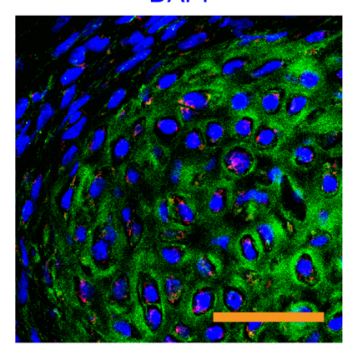

H\&E staining

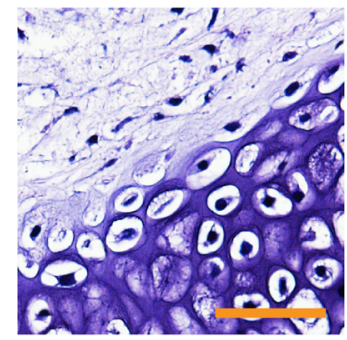

Human mitochondria/

DAPI

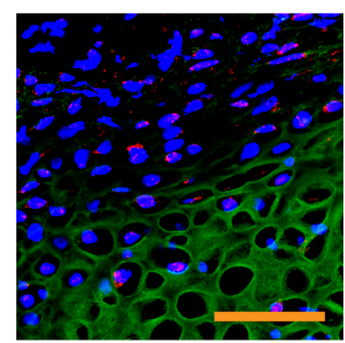

Mesoderm

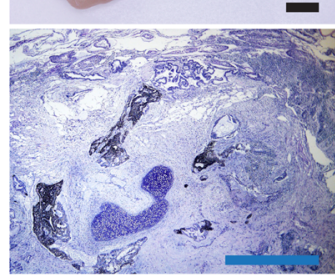

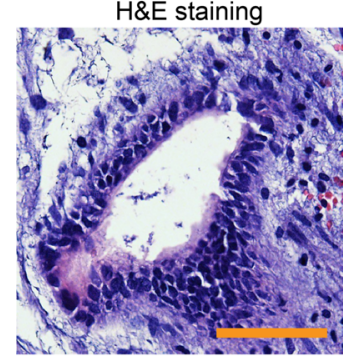

Human mitochondria/

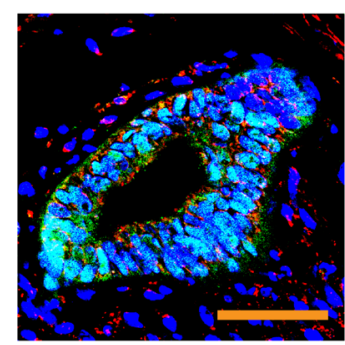

H\&E staining

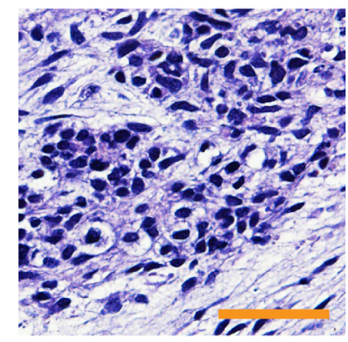

Human mitochondria/

FOXA2/DAPI

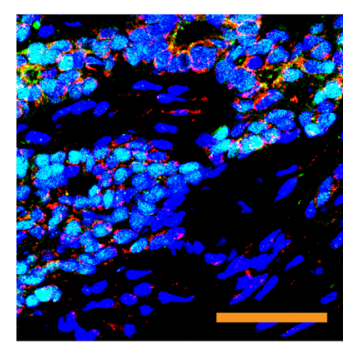

Endoderm 
Figure 8. Intratesticular implantation of CDKN2B-KD FReP cells in Fox Chase SCID Beige mice results in teratoma formation. (A) Gross appearance and histological evaluation (H\&E staining) of adult Fox Chase SCID Beige mouse testes with intratesticular implantation of $1 \times 10^{6}$ cells were assessed 4 months after implantation. All implanted mouse testes are shown in Supplemental Figure 8 ( $n=10$ mice per group). (B) Teratoma formation in CDKN2B-KD FReP cell-implanted animals was evaluated by a pathologist and confirmed by immunofluorescent staining; teratoma formation from retrovirus-mediated BJ-iPSC-implanted animals (Figure $4 \mathrm{~A}$ ) was used as a positive control. Three germ layers are clearly identified by ectoderm - pigmented cells; mesoderm cartilage with type II collagen staining; and endoderm gland with the definitive endoderm marker forkhead box A2 (FOXA2) staining. Scale bars: $5 \mathrm{~mm}$ (black), $1 \mathrm{~mm}$ (blue), or $50 \mu \mathrm{m}$ (orange).

derived from the University of California, Santa Cruz, Human Genome version 19 (hg19) (90). The results were submitted to the Gene Expression Omnibus (GEO GSE104830). Differentially expressed genes (fold change $\geq 2$ ) between FReP cells and BJ-derived iPSCs that were generated without genome integration (GEO SRR500985/6/7/8, ref. 23; and SRR1583694/5, ref. 24) were identified by the TopHatCufflinks package (25) through the Galaxy platform (26) using the default settings. DAVID Bioinformatics Resource 6.8 was used for functional annotation $(27,91)$. The differentially expressed genes that aligned with human proto-oncogenes and tumor suppressor genes, listed in the UniProt database (28), were displayed graphically by the package pheatmap (version 1.0.8) in $\mathrm{R}$ (version 3.4.1) (92).

Western blot. Cells were lysed using Pierce RIPA buffer supplemented with Halt Protease and Phosphatase Inhibitor Cocktail (Thermo Fisher Scientific). Western blotting was performed with antibodies against CDKN2B (catalog ab53034, Abcam) and GAPDH (product 5174S, clone D16H11, Cell Signaling Technology) to confirm the efficiency of CDKN2B KD at the protein level. Pierce ECL Western Blotting Substrate (Thermo Fisher Scientific) was used for development.

Intramuscular implantation animal model. Two-month-old male SCID mice (Charles River Laboratories) were anesthetized using inhaled $2 \%$ isoflurane and oxygen $(1.5 \mathrm{~L} / \mathrm{min})$ and were maintained in an oxygen flow environment upon awakening. A $0.5-\mathrm{cm}$ incision was made in the skin covering the left TA muscle, and $5 \times 10^{5}$ cells without premyogenic differentiation in vitro were slowly injected into the TA muscle. The fascia was then sutured in a simple continuous pattern, and the skin was closed separately using 4-0 Vicryl sutures (Ethicon Inc.) in a subcuticular pattern. Animals were euthanized 6 weeks after implantation. Both right (control) and left (implanted) TA muscles were harvested and weighed before fixation. The muscle masses of the left (implanted) and right (control) TA muscle of each mouse were compared to minimize the variation between individual animals.

Intratesticular teratoma formation assay. Since there are no detailed guidelines for tumorigenicity testing of cell-based therapeutic products, based on the protocols described in previous reports (13, 93), $1 \times 10^{6}$ cells in $30 \mu \mathrm{L}$ Matrigel hESC-qualified Matrix (BD Biosciences) were injected into the right testes of 2-month-old adult male Fox Chase SCID Beige mice (Charles River Laboratories). Fox Chase SCID Beige mice were used because they possess the autosomal recessive mutations SCID (Prkd ssid; resulting in a lack of functional T and B lymphocytes) and beige (Lyst ${ }^{\text {bg }}$; resulting in cytotoxic $\mathrm{T}$ cell and macrophage defects as well as selective impairment of NK cell functions)
(94). In compliance with the WHO recommendation (20), 10 animals were evaluated in each group. Tissues were harvested 4 months after implantation for gross appearance and histological assessments; however, teratomas were harvested before the 4-month endpoint when the animals' behaviors were affected $(13,93)$. Photos of harvested tissues were documented by an Olympus SZX12 microscope coupled with a DF PLAPO 1.2× PF object lens (Olympus) or a Canon EOS Rebel XSI camera coupled with an EF-S 60-mm lens and an MR-14EX Macro Ring Lite flashlight (Canon). Teratoma formation was assessed in a double-blind fashion. Following fixation, the teratomas were sectioned and stained with $\mathrm{H} \& \mathrm{E}$, and then analyzed by a pathologist to examine the presence of cells from each of the 3 germ layers.

Histological and immunological staining. Tissues were fixed in $4 \%$ paraformaldehyde (MilliporeSigma) for 24 hours, followed by dehydration and paraffin embedding. Five-micrometer sections were used for histological analysis with $\mathrm{H} \& \mathrm{E}$ and immunological staining. An antibody against ACTA1 (catalog ab28052, clone Alpha Sr-1, Abcam) was used to confirm myogenic differentiation, while antibodies against human MHC class I (catalog sc-25619, Santa Cruz Biotechnology) and human mitochondria (catalog MAB1273, clone 113-1, MilliporeSigma) were used for tracking human cells. In tissue that formed a teratoma, FOXA2 (catalog ab40874, Abcam) and type II collagen (catalog ab34712, Abcam) were used as markers of definitive endoderm-derivative and chondrocyte (mesenchymal/ mesoderm-derivative) cells, respectively. DAPI was used for nuclear counterstaining.

Statistics. All statistical analyses were conducted in consultation with the UCLA Statistical Biomathematical Consulting Clinic. Statistical analysis was computed by OriginPro 8 (OriginLab). Parametric data were compared by 1-way ANOVA and 2-sample $t$ tests (1-tailed), while 1-tailed Mann-Whitney and Kruskal-Wallis ANOVA tests were used for nonparametric data. For all data presented in this article, $P<$ $\left.0.05{ }^{*}\right)$ was considered a suggestive difference, while $\left.P<0.005{ }^{(* *}\right)$ was recognized as a statistically significant difference (95).

Study approval. All animal surgeries were performed under institutionally approved protocols provided by Chancellor's Animal Research Committee at UCLA (protocol 2012-119).

Data and materials availability. All data generated or analyzed during this study are included in this article (and its supplementary information files). The RNA-Seq data of FReP cells were submitted to the NIH Gene Expression Omnibus (GEO GSE104830).

\section{Author contributions}

ZZ conceived and designed the study. ZZ, CL, PY, WJ, XP, and $\mathrm{ZM}$ performed the in vitro and in vivo experiments, data interpretation, and statistical analysis, except for the following: $\mathrm{PH}$, GXC, and JKK performed the animal surgeries. XZ performed the pathological analysis of the teratomas. ZZ and CL wrote and revised the manuscript. $\mathrm{EAB}, \mathrm{CSH}$, and EC edited and proofread the manuscript. ZZ, KT, and CS supervised the entire study and approved the manuscript. All authors reviewed the final manuscript. The authorship order of the co-first authors was determined by flipping a coin.

\section{Acknowledgments}

We thank Guopin Fan (David Geffen School of Medicine, UCLA) for providing the BJ-iPSCs, and Matthew Schibler (Cal- 
ifornia NanoSystems Institute Advanced Light Microscopy/ Spectroscopy Shared Resource Facility, UCLA) for his assistance with the confocal laser scanning microscopy (CLSM) analysis. This study was supported by a Faculty seed grant from the UCLA School of Dentistry, the Plastic Surgery Foundation (2013 National Endowment for Plastic Surgery 269698), an $\mathrm{NIH} /$ National Center for Advancing Translational Sciences UCLA CTSI Grant (UL1TR001881), and the International S\&T Cooperation Program of China (grant 2013DFB30360). CLSM was performed at the Center for NanoScience Institute Advanced Light Microscopy/Spectroscopy Shared Resource Facility at UCLA.
Address correspondence to: Zhong Zheng, Division of Growth and Development, School of Dentistry, UCLA, 675 Charles E. Young Drive South, MRL 2641, Los Angeles, California 90095, USA. Phone: 310.206.5646; Email: zzheng@dentistry.ucla.edu. Or to: Kang Ting, Division of Growth and Development, School of Dentistry, UCLA, 10833 Le Conte Avenue, Box 951668, Los Angeles, California 90095, USA. Phone: 310.825.4384; Email: kting@ dentistry.ucla.edu. Or to: Chia Soo, Division of Plastic and Reconstructive Surgery and Department of Orthopaedic Surgery and the Orthopaedic Hospital Research Center, UCLA, 675 Charles E. Young Drive South, MRL 2641A, Los Angeles, California 90095, USA. Phone: 310.206.7783; Email: bsoo@ucla.edu.
1. Neves J, Sousa-Victor P, Jasper H. Rejuvenating strategies for stem cell-based therapies in aging. Cell Stem Cell. 2017;20(2):161-175.

2. Lye KL, Nordin N, Vidyadaran S, Thilakavathy K. Mesenchymal stem cells: from stem cells to sarcomas. Cell Biol Int. 2016;40(6):610-618.

3. Akimoto K, et al. Umbilical cord blood-derived mesenchymal stem cells inhibit, but adipose tissue-derived mesenchymal stem cells promote, glioblastoma multiforme proliferation. Stem Cells Dev. 2013;22(9):1370-1386.

4. Mason C, Dunnill P. A brief definition of regenerative medicine. Regen Med. 2008;3(1):1-5.

5 . Zheng Z, et al. Reprogramming of human fibroblasts into multipotent cells with a single ECM proteoglycan, fibromodulin. Biomaterials. 2012;33(24):5821-5831.

6. Madonna R, De Caterina R. In vitro neovasculogenic potential of resident adipose tissue precursors. Am J Physiol Cell Physiol. 2008;295(5):C1271-C1280.

7. Li CS, et al. Fibromodulin reprogrammed cells: a novel cell source for bone regeneration. Biomaterials. 2016;83:194-206.

8. Huard J, Li Y, Fu FH. Muscle injuries and repair: current trends in research. J Bone Joint Surg Am. 2002;84(5):822-832.

9. Turner NJ, Badylak SF. Regeneration of skeletal muscle. Cell Tissue Res. 2012;347(3):759-774.

10. Bentzinger CF, Wang YX, von Maltzahn J, Rudnicki MA. The emerging biology of muscle stem cells: implications for cell-based therapies. Bioessays. 2013;35(3):231-241.

11. Sirabella D, De Angelis L, Berghella L. Sources for skeletal muscle repair: from satellite cells to reprogramming. JCachexia Sarcopenia Muscle. 2013;4(2):125-136

12. Shadrin IY, Khodabukus A, Bursac N. Striated muscle function, regeneration, and repair. Cell Mol Life Sci. 2016;73(22):4175-4202.

13. Lensch MW, Ince TA. Assaying pluripotency via teratoma formation. In: Stein GS, Borowski M, Luong MX, Shi MJ, Smith KP, Vazquez P, eds. Human Stem Cell Technology and Biology: A research guide and laboratory manual. Hoboken, New Jersey, USA: Wiley-Blackwell; 2011:217-233.

14. Le T, Kim KP, Fan G, Faull KF. A sensitive mass spectrometry method for simultaneous quantification of DNA methylation and hydroxymethylation levels in biological samples. Anal Biochem. 2011;412(2):203-209.

15. Chamberlain JS, Jaynes JB, Hauschka SD.
Regulation of creatine kinase induction in differentiating mouse myoblasts. Mol Cell Biol. 1985;5(3):484-492.

16. Panda AC, et al. RNA-binding protein AUF1 promotes myogenesis by regulating MEF2C expression levels. Mol Cell Biol. 2014;34(16):3106-3119.

17. Ben-David U, Benvenisty N. The tumorigenicity of human embryonic and induced pluripotent stem cells. Nat Rev Cancer. 2011;11(4):268-277.

18. Tapia N, Schöler HR. p53 connects tumorigenesis and reprogramming to pluripotency. J Exp Med. 2010;207(10):2045-2048.

19. Menendez S, et al. Increased dosage of tumor suppressors limits the tumorigenicity of iPS cells without affecting their pluripotency. Aging Cell. 2012;11(1):41-50.

20. WHO. Recommendations for the evaluation of animal cell cultures as substrates for the manufacture of biological medicinal products for the characterization of cell banks: proposed replacement of Trs 878, annex 1. WHO website. https://www.who int/biologicals/Cell_Substrates_clean_version_18_ April.pdf. Accessed June 24, 2019.

21. Kamada M, Mitsui Y, Matsuo T, Takahashi T. Reversible transformation and de-differentiation of human cells derived from induced pluripotent stem cell teratomas. Hum Cell. 2016;29(1):1-9.

22. Shukla V, et al. ASXL3 is a novel pluripotency factor in human respiratory epithelial cells and a potential therapeutic target in small cell lung cancer. Cancer Res. 2017;77(22):6267-6281.

23. Yoshioka N, et al. Efficient generation of human iPSCs by a synthetic self-replicative RNA. Cell Stem Cell. 2013;13(2):246-254.

24. Johannesson B, et al. Comparable frequencies of coding mutations and loss of imprinting in human pluripotent cells derived by nuclear transfer and defined factors. Cell Stem Cell. 2014;15(5):634-642.

25. Trapnell C, et al. Differential gene and transcript expression analysis of RNA-seq experiments with TopHat and Cufflinks. Nat Protoc. 2012;7(3):562-578.

26. Afgan E, et al. The Galaxy platform for accessible, reproducible and collaborative biomedical analyses: 2016 update. Nucleic Acids Res. 2016;44(W1):W3-W10.

27. Huang DW, Sherman BT, Lempicki RA. Systematic and integrative analysis of large gene lists using DAVID bioinformatics resources. Nat Protoc. 2009;4(1):44-57.

28. The UniProt Consortium. UniProt: the univer- sal protein knowledgebase. Nucleic Acids Res 2017;45(D1):D158-D169.

29. Nakagawa M, Takizawa N, Narita M, Ichisaka T, Yamanaka S. Promotion of direct reprogramming by transformation-deficient Myc. Proc Natl Acad Sci U S A. 2010;107(32):14152-14157.

30. Hanna J, et al. Direct cell reprogramming is a stochastic process amenable to acceleration. Nature. 2009;462(7273):595-601.

31. Szklarczyk D, et al. The STRING database in 2017: quality-controlled protein-protein association networks, made broadly accessible. Nucleic Acids Res. 2017;45(D1):D362-D368.

32. el-Deiry WS, et al. WAF1, a potential mediator of p53 tumor suppression. Cell. 1993;75(4):817-825.

33. Okita K, Yamanaka S. Induced pluripotent stem cells: opportunities and challenges. Philos Trans $R$ Soc Lond B Biol Sci. 2011;366(1575):2198-2207.

34. Molchadsky A, Rivlin N, Brosh R, Rotter V, Sarig R. p53 is balancing development, differentiation and de-differentiation to assure cancer prevention. Carcinogenesis. 2010;31(9):1501-1508.

35. Ebrahimi B. Reprogramming barriers and enhancers: strategies to enhance the efficiency and kinetics of induced pluripotency. Cell Regen (Lond). 2015;4:10.

36. Hong H, et al. Suppression of induced pluripotent stem cell generation by the p53-p21 pathway. Nature. 2009;460(7259):1132-1135.

37. Moriguchi H, Chung RT, Sato C. Tumorigenicity of human induced pluripotent stem cells depends on the balance of gene expression between p21 and p53. Hepatology. 2010;51(3):1088-1089.

38. García-Cao I, et al. "Super p53" mice exhibit enhanced DNA damage response, are tumor resistant and age normally. EMBO J. 2002;21(22):6225-6235.

39. Matheu A, et al. Increased gene dosage of Ink $4 \mathrm{a} /$ Arf results in cancer resistance and normal aging. Genes Dev. 2004;18(22):2736-2746.

40. Mikkelsen TS, et al. Dissecting direct reprogramming through integrative genomic analysis. Nature. 2008;454(7200):49-55.

41. Malecki M. 'Above all, do no harm': safeguarding pluripotent stem cell therapy against iatrogenic tumorigenesis. Stem Cell Res Ther. 2014;5(3):73.

42. García-Castro J, Trigueros C, Madrenas J, PérezSimón JA, Rodriguez R, Menendez P. Mesenchymal stem cells and their use as cell replacement therapy and disease modelling tool. J Cell Mol Med. 2008;12(6B):2552-2565.

43. Lu SH, Wei CF, Yang AH, Chancellor MB, 
Wang LS, Chen KK. Isolation and characterization of human muscle-derived cells. Urology. 2009;74(2):440-445.

44. Stolzing A, Jones E, McGonagle D, Scutt A. Agerelated changes in human bone marrow-derived mesenchymal stem cells: consequences for cell therapies. Mech Ageing Dev. 2008;129(3):163-173.

45. Terranova C. Death after liposuction. In: Shiffman MA, Giuseppe AD, eds. Liposuction: Principles and Practice. Heidelberg, Berlin, Germany: Springer Verlag Berlin Heidelberg; 2016:825-832.

46. Phinney DG, Prockop DJ. Concise review: mesenchymal stem/multipotent stromal cells: the state of transdifferentiation and modes of tissue repair current views. Stem Cells. 2007;25(11):2896-2902.

47. Kean TJ, Lin P, Caplan AI, Dennis JE. MSCs: delivery routes and engraftment, cell-targeting strategies, and immune modulation. Stem Cells Int. 2013;2013:732742.

48. Murphy MB, Moncivais K, Caplan AI. Mesenchymal stem cells: environmentally responsive therapeutics for regenerative medicine. Exp Mol Med. 2013;45:e54.

49. Rohban R, Pieber TR. Mesenchymal stem and progenitor cells in regeneration: tissue specificity and regenerative potential. Stem Cells Int. 2017;2017:5173732.

50. Caplan AI. New MSC: MSCs as pericytes are sentinels and gatekeepers. JOrthop Res. 2017;35(6):1151-1159.

51. Hofer HR, Tuan RS. Secreted trophic factors of mesenchymal stem cells support neurovascular and musculoskeletal therapies. Stem Cell Res Ther. 2016;7(1):131.

52. Kocan B, Maziarz A, Tabarkiewicz J, Ochiya T, Banaś-Ząbczyk A. Trophic activity and phenotype of adipose tissue-derived mesenchymal stem cells as a background of their regenerative potential. Stem Cells Int. 2017;2017:1653254.

53. Omel'chenko DO, Rzhaninova AA, Gol'dshteĭn DV. [Comparative transcriptome pairwise analysis of spontaneously transformed multipotent stromal cells from human adipose tissue]. Genetika. 2014;50(1):106-115.

54. Pan Q, et al. Detection of spontaneous tumorigenic transformation during culture expansion of human mesenchymal stromal cells. Exp Biol Med (Maywood). 2014;239(1):105-115.

55. Ren G, et al. CCR2-dependent recruitment of macrophages by tumor-educated mesenchymal stromal cells promotes tumor development and is mimicked by TNF $\alpha$. Cell Stem Cell. 2012;11(6):812-824.

56. Furno EL, Laan Svd, Maiorano D. Genomic instability of pluripotent stem cells: origin and consequences. In: Tomizawa M, ed. Pluripotent Stem Cells: From the Bench to the Clinic. London, United Kingdom: IntechOpen; 2016:674-689.

57. Mandai M, et al. Autologous induced stemcell-derived retinal cells for macular degeneration. N Engl J Med. 2017;376(11):1038-1046.

58. Cao S, et al. Chromatin accessibility dynamics during chemical induction of pluripotency. Cell Stem Cell. 2018;22(4):529-542.e5.
59. Moore KA, Lemischka IR. Stem cells and their niches. Science. 2006;311(5769):1880-1885.

60. Hansis C, Barreto G, Maltry N, Niehrs C. Nuclear reprogramming of human somatic cells by xenopus egg extract requires BRG1. Curr Biol. 2004;14(16):1475-1480.

61. Zhu XQ, et al. Transient in vitro epigenetic reprogramming of skin fibroblasts into multipotent cells. Biomaterials. 2010;31(10):2779-2787.

62. Taranger CK, Noer A, Sørensen AL, Håkelien $\mathrm{AM}$, Boquest AC, Collas P. Induction of dedifferentiation, genomewide transcriptional programming, and epigenetic reprogramming by extracts of carcinoma and embryonic stem cells. Mol Biol Cell. 2005;16(12):5719-5735.

63. Kim DW, Staples M, Shinozuka K, Pantcheva P, Kang SD, Borlongan CV. Wharton's jelly-derived mesenchymal stem cells: phenotypic characterization and optimizing their therapeutic potential for clinical applications. Int J Mol Sci. 2013;14(6):11692-11712

64. Kalaszczynska I, Ferdyn K. Wharton's jelly derived mesenchymal stem cells: future of regenerative medicine? Recent findings and clinical significance. Biomed Res Int. 2015;2015:430847.

65. Mauro A, et al. Isolation, characterization, and in vitro differentiation of ovine amniotic stem cells. Vet Res Commun. 2010;34(suppl 1):S25-S28.

66. Marmotti A, et al. Allogeneic umbilical cordderived mesenchymal stem cells as a potential source for cartilage and bone regeneration: an in vitro study. Stem Cells Int. 2017;2017:1732094.

67. Davies JE, Walker JT, Keating A. Concise review: Wharton's jelly: the rich, but enigmatic, source of mesenchymal stromal cells. Stem Cells Transl Med. 2017;6(7):1620-1630.

68. Soo C, Beanes S, Dang C, Zhang X, Ting K. Fibromodulin, a TGF- $\beta$ modulator, promotes scarless fetal repair. Surgical Forum. 2001;52:578-581.

69. Zheng Z, et al. Fibromodulin is essential for fetal-type scarless cutaneous wound healing. Am J Pathol. 2016;186(11):2824-2832.

70. Zheng Z, et al. Fibromodulin reduces scar formation in adult cutaneous wounds by eliciting a fetal-like phenotype. Signal Transduct Target Ther. 2017;2:17050.

71. Bi Y, et al. Identification of tendon stem/progenitor cells and the role of the extracellular matrix in their niche. Nat Med. 2007;13(10):1219-1227.

72. Li CJ, et al. Long noncoding RNA Bmncr regulates mesenchymal stem cell fate during skeletal aging. J Clin Invest. 2018;128(12):5251-5266.

73. Fisch SC, et al. Pluripotent nontumorigenic multilineage differentiating stress enduring cells (Muse cells): a seven-year retrospective. Stem Cell Res Ther. 2017;8(1):227.

74. Hannon GJ, Beach D. p15INK4B is a potential effector of TGF-beta-induced cell cycle arrest. Nature. 1994;371(6494):257-261.

75. De Braekeleer M, Douet-Guilbert N, De Braekeleer E. Prognostic impact of p15 gene aberrations in acute leukemia. Leuk Lymphoma. 2017;58(2):257-265
76. $\mathrm{Li} \mathrm{H}$, et al. The Ink4/Arf locus is a barrier for iPS cell reprogramming. Nature. 2009;460(7259):1136-1139.

77. Mali $\mathrm{P}$, et al. RNA-guided human genome engineering via Cas9. Science. 2013;339(6121):823-826.

78. Yang L, et al. Optimization of scarless human stem cell genome editing. Nucleic Acids Res. 2013;41(19):9049-9061.

79. Li M, Suzuki K, Kim NY, Liu GH, Izpisua Belmonte JC. A cut above the rest: targeted genome editing technologies in human pluripotent stem cells. J Biol Chem. 2014;289(8):4594-4599.

80. Ihry RJ, et al. p53 inhibits CRISPR-Cas9 engineering in human pluripotent stem cells. Nat Med. 2018;24(7):939-946.

81. Tsai SQ, Joung JK. Defining and improving the genome-wide specificities of CRISPR-Cas9 nucleases. Nat Rev Genet. 2016;17(5):300-312.

82. [No authors listed]. Keep off-target effects in focus. Nat Med. 2018;24(8):1081.

83. Yamanaka S. A fresh look at iPS cells. Cell. 2009;137(1):13-17.

84. Zheng Z, et al. Delayed wound closure in fibromodulin-deficient mice is associated with increased TGF- $\beta 3$ signaling. J Invest Dermatol. 2011;131(3):769-778.

85. Crisan M, et al. A perivascular origin for mesenchymal stem cells in multiple human organs. Cell Stem Cell. 2008;3(3):301-313.

86. Singh Roy N, Nakano T, Xuing L, Kang J, Nedergaard M, Goldman SA. Enhancer-specified GFP based FACS purification of human spinal motor neurons from embryonic stem cells. Exp Neurol. 2005;196(2):224-234.

87. Nizzardo M, et al. Human motor neuron generation from embryonic stem cells and induced pluripotent stem cells. Cell Mol Life Sci. 2010;67(22):3837-3847.

88. D’Amour KA, Agulnick AD, Eliazer S, Kelly OG, Kroon E, Baetge EE. Efficient differentiation of human embryonic stem cells to definitive endoderm. Nat Biotechnol. 2005;23(12):1534-1541.

89. Huangfu D, et al. Induction of pluripotent stem cells from primary human fibroblasts with only Oct4 and Sox2. Nat Biotechnol. 2008;26(11):1269-1275.

90. Kent WJ, et al. The human genome browser at UCSC. Genome Res. 2002;12(6):996-1006.

91. Huang da W, Sherman BT, Lempicki RA. Bioinformatics enrichment tools: paths toward the comprehensive functional analysis of large gene lists. Nucleic Acids Res. 2009;37(1):1-13.

92. R Core Team. 2014. R: a language and environment for statistical computing. R Foundation for Statistical Computing, Vienna, Austria. http:// www.R-project.org/.

93. Peterson SE, et al. Teratoma generation in the testis capsule. J Vis Exp. 2011;57(57):e3177.

94. Frandsen TL, Holst-Hansen C, Brunner N. Tumorigenicity assays. In: Encyclopedia of Life Sciences. Chichester, United Kingdom: John Wiley \& Sons; 2002.

95. Benjamin DJ, et al. Redefine statistical significance. Nat Hum Behav. 2018;2(1):6-10. 